\title{
Palynology of the marine Jurassic formations in the Vardekløft ravine, Jameson Land, East Greenland
}

\author{
JENS JøRGEN LUND and KAJ RAUNSGAARD PEDERSEN
}

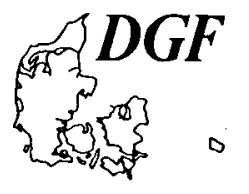

\begin{abstract}
Lund, J. J. and Pedersen, K. Raunsgaard: Palynology of the marine Jurassic formations in the Vardekløft ravine, Jameson Land, East Greenland. Bull. geol. Soc. Denmark, vol. 33, pp. 371-399, Copenhagen, February, 28th, 1985.

The microfloras of the marine Neill Klinter, Vardekløft, and lower Hareelv Formations have been investigated from exposures in the Vardekløft ravine at Hurry Inlet, eastern Jameson Land. The samples have yielded abundant spores and pollen as well as dinoflagellate cysts. Based on the occurrence of spores and pollen four microfloral assemblages (A-D) have been established. Biostratigraphic correlations based on the dinoflagellate occurrences as well, have been proposed by comparison with microfloras of Europe.

Assemblage A and B of the Neill Klinter Formation have been correlated to (Late) Pliensbachian to Early Toarcian. The typical assemblage $\mathrm{C}$ microfloras are restricted to the Vardekløft Formation and is correlated to the Middle-Late Bajocian at the base of the formation and to the (Early) Callovian at the top. The assemblage D has been recovered from the lower Hareelv Formation. Based on the dinoflagellate cysts the deepest samples available from this formation may be correlated to the Oxfordian cordatum(-plicatilis) Zone while the upper part of the section in Vardekløft is referred to the Early Kimmeridgian.

The composition of the microfloras indicates marine to brackish palaeoenvironments with considerable variations in the terrestrial derived palynomorph groups.

Comparison with age equivalent microfloras of Svalbard and Andøya, Northern Norway, and the Middle Europe indicates the stratigraphical distribution of some spores to be palaeolatitudinally controlled.
\end{abstract}

Jens Jørgen Lund, Deutsche Texaco AG, D-3109 Wietze, W. Germany. Kaj Raunsgaard Pedersen, Geological Institute, University of Aarhus, DK-8000 Arhus C, Denmark, October 9th, 1984.

The eastern part of Jameson Land exposes a sequence of Upper Triassic to Upper Jurassic sediments about $700 \mathrm{~m}$ thick with a lower limnic and a thick upper marine series.

The limnic plant bearing Kap Stewart Formation in the lower part of the profiles was palynologically investigated by Pedersen \& Lund (1980) and was divided into two microflora zones with subzones of Lower Rhaetian and Hettangian age respectively. The present work is a continuation of our palynological profiles in the central part of the Hurry Inlet area. The marine series here investigated from the Vardekløft ravine is about $500 \mathrm{~m}$ thick and includes the Neill Klinter and Vardekløft Formations and the lower part of the Hareelv Formation. From these formations four spore-pollen assemblages are recognized with ages ranging from Pliensbachian to Early Kimmeridgian.

In connection with the geological mapping of Jameson Land and Scoresby Land a modified formation and member concept was proposed
(Surlyk, Callomon, Bromley \& Birkelund, 1973). The geological age of these units was determined by new and previously known fossil faunas, particularly ammonites, and is here related to the palynological results.

The material for the palynological investigation was collected by K. Raunsgaard Pedersen.

\section{Geology of the Vardekløft area}

Vardekløft is a rather large ravine in the central part of the Neill Klinter cliff which faces Hurry Inlet (fig. 1, 2, 3). It extends from about $150 \mathrm{~m}$ to about $700 \mathrm{~m}$ above sea level, intersecting the Kap Stewart, Neill Klinter, Vardekløft, and Hareelv Formations. Due to heavy scree, the sediments are generally poorly exposed. The best exposures are on the steeper slopes facing north.

The limnic Kap Stewart Formation as exposed in the lower profiles in Vardekløft is $80 \mathrm{~m}$ thick. The formation is dominated by arkosic sand- 


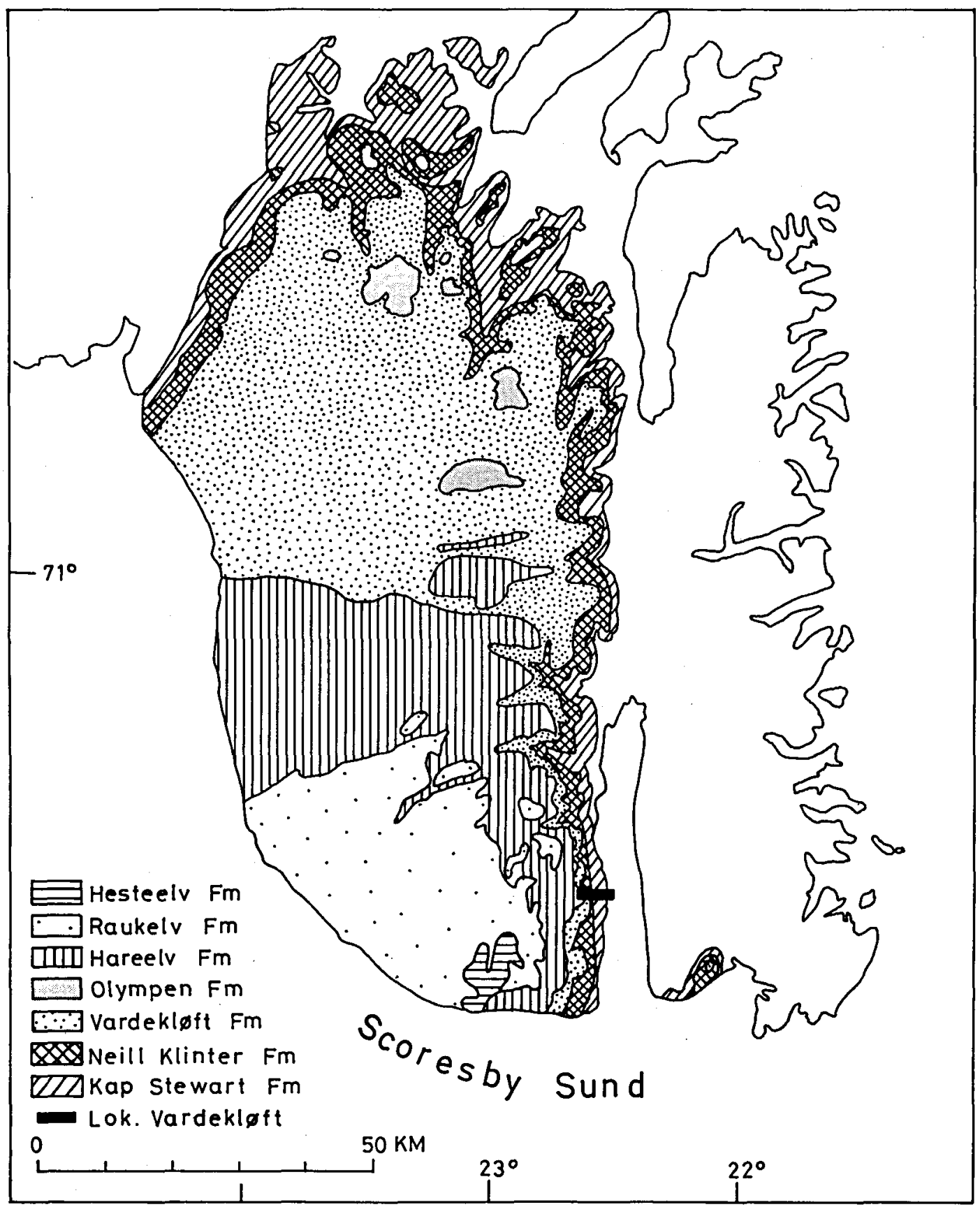

Fig. 1. Simplified geological map of the Jameson Land area showing distribution of Jurassic-Lower Cretaceous formations and the position of the Vardekløft ravine (after Surlyk et al., 1973).

stones with shale intercalations occurring in particular in the lower part. Plant megafossils have been described by Harris (1937) from six layers four of which represent the Lepidopteris Zone and the remaining two the Thaumatopteris Zone.
The superposed marine Neill Klinter Formation is about $200 \mathrm{~m}$ thick and dominated by sandstones with few shale intercalations. Based on sedimentary structures and common trace fossils the depositional environment has been interpre- 
ted as low to high energy near shore marine (Surlyk, Bromley, Asgaard \& Pedersen, 1971; Surlyk et al., 1973). The Neill Klinter Formation has been subdivided into three members, of which the lower (Rævekløft Member) contains a very rich fauna referred to the Uptonia jamesoni Zone of Pliensbachian age (Surlyk et al., 1973). The hiatus between the Kap Stewart Formation and Neill Klinter Formation most probably represents either the entire Sinemurian or at least the upper part of Sinemurian (Pedersen \& Lund, 1980). The middle member (Gule Horn Member) contains only trace fossils, but in the upper member (Ostreaelv Member) fossils indicating a Toarcian age have been collected (Surlyk et al., 1973). The Neill Klinter Formation is intruded by three large sills which together with the coarse sediments cause several levels to be barren of palynomorphs.

The overlying Vardekløft Formation (of which the ravine is the type locality) comprises about $250 \mathrm{~m}$ of sandy and clayey sediments. The formation is generally covered by scree especially on the middle of the three members. The lower Sorthat Member consists of black-grey shale with concretions (about $60 \mathrm{~m}$ ). Plant fragments and marine fossils have been recorded, but as none are age diagnostic the exact correlation of this member is uncertain. From the ages of the sediments below and above, the Sorthat Member is considered to be of Bajocian age with a hiatus below comprising the Upper Toarcian (?) and Aalenian (Surlyk et al., 1973).

The middle Pelion Member is a light coloured sandstone which in the Vardekløft area is less than $50 \mathrm{~m}$ thick and badly exposed. In the Hurry Inlet area the sandstone has yielded fossils of Upper Bajocian age (Surlyk et al., 1973).

The upper member of the Vardekløft Formation (Fossilbjerg Member) is about $100 \mathrm{~m}$ thick. The fine grained sandstone and grey silty shale contain numerous concretions which often contain fossil wood. Ammonites and belemnites of Bathonian to Middle Callovian age are also common in certain horizons. Two major hiati were recognized at this stratigraphic level in the Hurry Inlet area, one occurring just above the Pelion Member and the other below a soft grey shale in the upper part of Fossilbjerg Member (Surlyk et al., 1973). The depositional environment of the three members of Vardekløft Formation is interpreted as low energy offshore marine for the Sorthat Member, high energy sublittoral marine for the Pelion Member and low energy marine for the Fossilbjerg Member (Surlyk et al., 1973).

The lower part of the Hareelv Formation forms the plateau at about $700 \mathrm{~m}$ above sea and occurs in the upper part of the Vardekløft ravine. The Hareelv Formation comprises a sequence of alternating layers or lenses of yellow sandstone and black soft shales which vary laterally. Only the lower $50 \mathrm{~m}$ Hareelv Formation (which can attain a thickness of $200 \mathrm{~m}$ ) is represented in the Vardekløft area. Invertebrate fossils from the lower part of this formation indicate an early Late Oxfordian age. On basis of ammonite occurrence a hiatus between the Vardekløft and the Hareelv Formation thus comprises Middle? and Late Callovian together with Early (and Middle) Oxfordian. The depositional environment for the Hareelv Formation is interpreted as marine far offshore with gravity transported sands (Surlyk et al., 1973).

\section{Sample preparation}

The palynological samples investigated (fig. 4) were of rather variable quality as was the case with samples from the underlying Kap Stewart Formation (Pedersen \& Lund, 1980). The siltysandy samples in particular yielded rather poorly preserved palynomorphs. The preparation technique utilized includes treatment in $10 \% \mathrm{HCl}$ followed by concentrated $\mathrm{HF}$ at $100^{\circ} \mathrm{C}$ in $2+2 \mathrm{~h}$ and $10 \% \mathrm{HCl}$ at $100^{\circ} \mathrm{C}$. Separation in $\mathrm{ZnCl}_{2}$ s.g. 1.90 . Sievings on nylon filter $(10 \mu \mathrm{m})$ have been used after the HF treatment. Oxidation with Schulze mixture was employed from 0 to $5 \mathrm{~min}$. as was treatment with $5 \% \mathrm{KOH}$ for 0 to $5 \mathrm{~min}$. The organic residue is stored in silicon oil and glycerin.

The slides with the illustrated specimens and a list with stage coordinates for the Leitz Orthomat microscope are at the Geological Institute, University of Aarhus, and will be permanently deposited in the Geological Museum, Copenhagen under MGUH no. 16587 to 16653 .

All sample no. quoted in the text are the last two digits of GGU nos. 142820 to 142858 . 

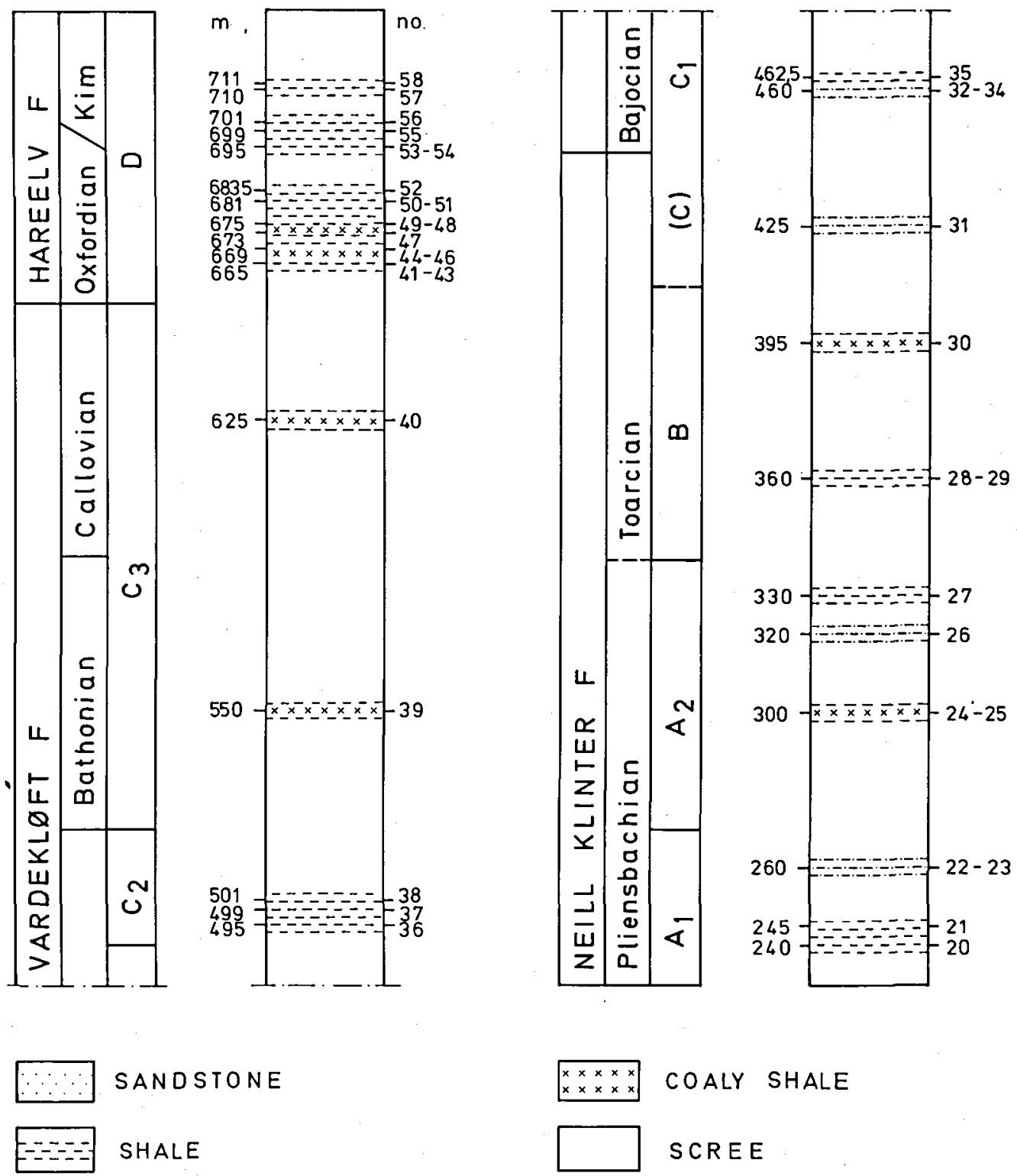

SANDSTONE

$x \times x \times x$

$\times \times \times \times \times$

COALY SHALE

SHALE

SCREE

Fig. 4. Generalized section of the marine formations from the Vardekløft ravine. The figures to the left indicate meters above sea level (altimeter readings) and those to the right are the last two digits of GGU no. 142820 to 142858 .

\section{Systematic palynology}

The morphosystematic and systematic at genus and species level are generally in accordance with Pedersen and Lund (1980). Authors referred to only in the systematics on genus and higher sys- tematic levels are omitted in the references. Species and genus names of dinoflagellate cysts are used in accordance with Riley and Fenton (1982) and Stover and Evitt (1978). 


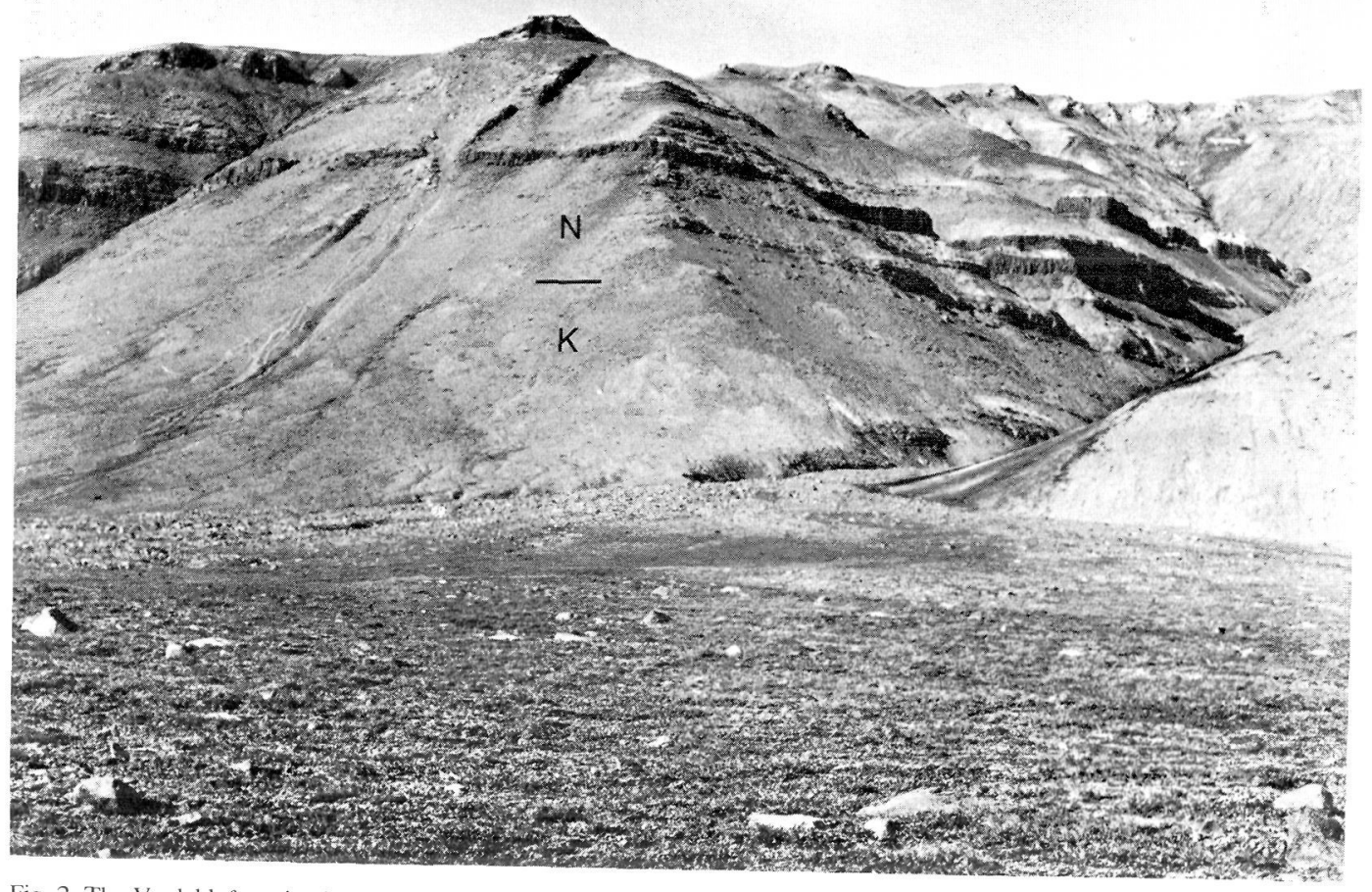

Fig. 2. The Vardekløft ravine in the cliff of Neill Klinter, eastern Jameson Land. The lower exposures are in the Kap Stewart Formation $(\mathrm{K})$. The sills penetrate the Neill Klinter Formation $(\mathrm{N})$. The upper part of the ravine intersects the Vardekløft Formation and
the lower Hareelv Formation.

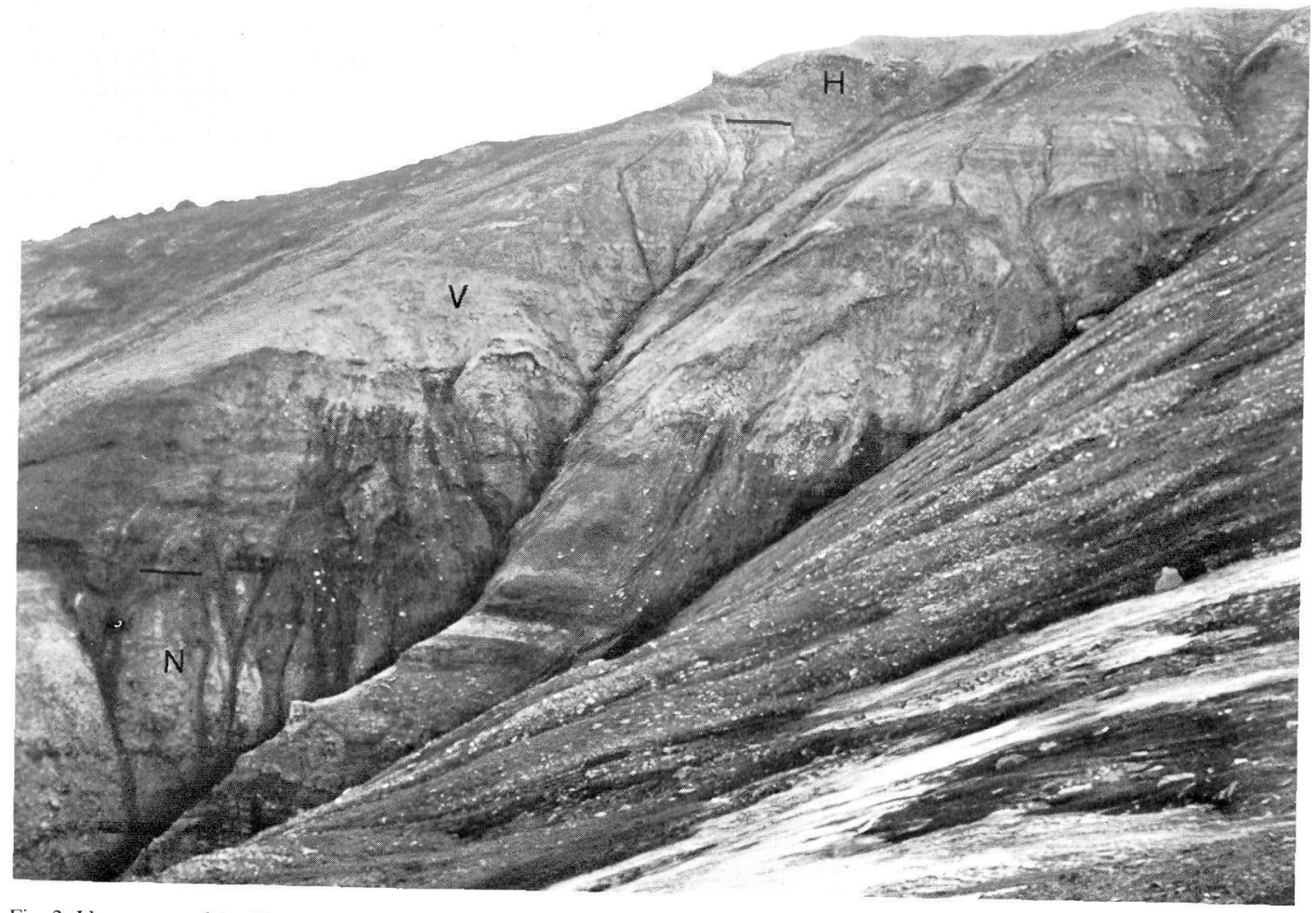

Fig. 3. Upper part of the Vardekløft ravine exposing the upper part of the Neill Klinter Formation (N), the Vardekløft Formation (V)
and the lower part of the Hareelv Formation $(\mathrm{H})$. 


\section{Spores and pollen}

Anteturma: Sporites H. Potonié

Turma: Triletes (Reinsch) Dettmann

Infraturma: Laevigati (Bennie \& Kidston) R. Potonié

Deltoidospora Miner 1935

Deltoidospora spp.

Assemblage A-D.

Deltoidospora minor (Couper) Pocock 1970

Pl. 1, fig. 1. Assemblage A1, C2, D.

Deltoidospora sp. 1

PI. 1, fig. 2. Assemblage D.

Remarks: This species is characterized by absence of contact-area thickenings and by its large size $(47-62 \mu \mathrm{m})$.

Gleicheniidites Ross 1949

Gleicheniidites conspiciendus (Bolkhovitina)

Krutzsch 1959

P1. 1, fig. 4. Assemblage D, base in sample 43, regularly present in sample 51 and higher.

Gleicheniidites senonicus Ross 1949

Pl. 1, fig. 3. Assemblage D, base in sample 49, rare.

Intrapunctisporis Krutzsch 1959

Intrapunctisporis toralis (Leschik) Lund 1977

Assemblage D.

Punctatisporites (Ibrahim 1933) Potonié \&

Kremp 1954

Punctatisporites major (Couper) Kedves \& Simoncsics 1964

Assemblage A.

Punctatisporites globosus (Leschik) Lund 1977

Assemblage A-C1.

Calamospora Schopf, Wilson \& Bentall 1944

Calamospora tener (Leschik) Mädler 1964

Assemblage $\mathrm{C} 1$.

Stereisporites Pflug 1953

Stereisporites spp.

Assemblage A, B, C, D, rare in assemblage D. Stereisporițes stereoides (Potonié \& Venitz) Pflug 1953
Assemblage A, ?C1.

Stereisporites cf. seebergensis E. Schulz 1966

Assemblage C3, D lower part.

Stereisporites cf. lunaris (Rogalska) Lund 1977

Assemblage A1.

Stereisporites cicatricosus (Rogalska) E. Schulz 1967

Pl. 1. fig. 5. Assemblage A1, C1, C3, D lower part.

Stereisporites aulosensis (E. Schulz) E. Schulz 1966

Assemblage C2, D middle part.

Infraturma: Apiculati (Bennie \& Kidston) R. Potonié

Conbaculatisporites Klaus 1960

Conbaculatisporites mesozoicus Klaus 1960

Assemblage A1, ?B, C3.

Neoraistrickia Potonié 1956

cf. Neoraistrickia sp.

Assemblage C3, D lower part.

Remarks: Size about $35 \mu \mathrm{m}$, baculae (and clavae) 2-3 $\mu \mathrm{m}$ high and $1-2 \mu \mathrm{m}$ in diameter. This species is intermediate between Conbaculatisporites and Neoraistrickia.

Baculatisporites Thomson \& Pflug 1953

Baculatisporites comaumensis (Cookson) Potonié 1956

Pl. 1, fig. 6. Assemblage A, B, C, D, frequent in assemblage $\mathrm{A} 1, \mathrm{C} 1$ and $\mathrm{C} 3$.

Baculatisporites wellmanii (Couper) Krutzsch 1959

Assemblage A1, C2, C3.

Baculatisporites sp. 1

Pl. 1, fig. 7. Assemblage A1, B, ?C1, C3.

Remarks: Characterized by its gemmate sculpture.

Baculatisporites sp. 2

Pl. 1, fig. 8. Assemblage A1, C, D (sample 58 only); acme in assemblage A1.

Remarks: Characterized by its triangular outline. aff. Baculatisporites sp. 3

Pl. 2, fig. 1. Assemblage A; acme in assemblage A1.

Explanation of plates

All photographs of spores and pollen (plates 1 to 6 ) are $\times 1000$. The photographs of dinoflagellate cysts (plates 7 to 8 ) are $\times 500$. Slide indication in brackets: GGU sample no., preparation no. and slide no.

MGUH no. is the collection no. of the Geological Museum, Copenhagen. 

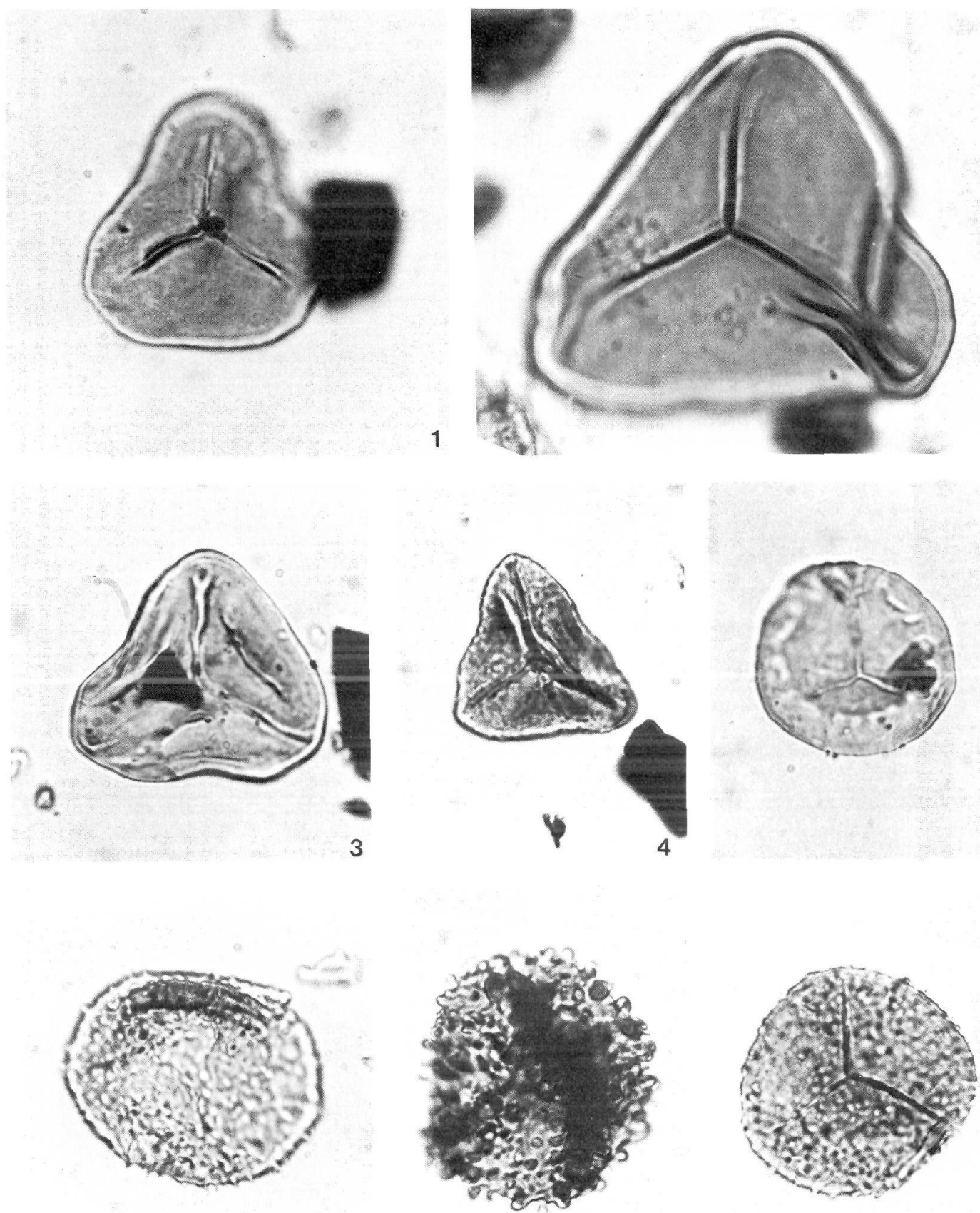

6

Plate 1. Fig. 1; Deltoidospora minor (Couper) Pocock. Hareelv F., (142851, 3-3). MGUH 16587. Fig. 2. Deltoidospora sp. 1. Hareelv F. (142852, 3-2). MGUH 16588. Fig. 3; Gleicheniidites senonicus Ross. Hareelv F., (142849, 3-1). MGUH 16589. Fig. 4; Gleicheniidites conspiciendus (Bolkhovitina) Krutzsch. Hareelv F., (142851, 3-3). MGUH 16590. Fig. 5; Stereisporites cicatricosus (Rogalska) E. Schulz. Vardekløft F., (142834, 4-5). MGUH 16591. Fig. 6; Baculatisporites comaumensis (Cookson) Potonié. Neill Klinter F. (142821, 3-3). MGUH 16592. Fig. 7; Baculatisporites sp. 1. Neill Klinter F., (142823, 3-1). MGUH 16593. Fig. 8; Baculatisporites sp. 2. Vardekløft F., (142834, 4-5). MGUH 16594. 
Remarks: This species is characterized by its large size $(60-80 \mu \mathrm{m})$ and the conate (to baculate) sculpture. The length of the laesura has not been observed.

Apiculatisporis Potonié \& Kremp 1956

Apiculatisporis ovalis (Nilsson) Norris 1965

Pl. 2, fig. 2. Assemblage A, C1, C3; acme in A1.

Trachysporites Nilsson 1958

Trachysporites fuscus Nilsson 1958

Pl. 2, fig. 3. Assemblage A1, C3, ?D.

Concavisporites Pflug 1953

Concavisporites granulosus Tralau 1968 sensu

Vigran \& Thusu 1975

Assemblage C1.

Remarks: The sculpture consists of partly fused baculae forming a rugulate sculpture.

Manumia Pocock 1970

Manumia cf. irregularis Pocock 1970

Pl. 2, fig. 4. Assemblage B, C2.

Leptolepidites Couper 1953

Leptolepidites major Couper 1958 .

Pl. 2, fig. 5. Assemblage C.

Leptolepidites bossus (Couper) E. Schulz 1967

Pl. 2, fig. 6. Assemblage B, C1.

Foraminisporis Krutzsch 1959

Foraminisporis jurassicus E. Schulz 1967

Assemblage A1.

Iraquispora Singh 1964

Iraquispora laevigata (Mädler) Lund 1977

Assemblage A2.

Iraquispora sp.

Assemblage A1.

Remarks: The spore is similar in size and shape to I. laevigata. It is similar to "Kyrtomisporites" gracilis Bjærke 1977 in its distal sculpture with radiating ridges.

Infraturma: Murornati Potonié \& Kremp

Lycopodiumsporites (Thiergart 1938) Delcourt \& Sprumont 1955

Lycopodiumsporites semimuris Danzé-Corsin \& Laveine 1963

Pl. 3, fig. 2. Assemblage C3.

Lycopodiumsporites spp.

Pl. 2, fig. 7, pl. 3, fig. 1. Assemblage A, C, D.

Remarks: The assemblages are thought to represent up to $\mathbf{1 0}$ species, but it has shown difficult to define usable species. Fairly well-defined forms/ types occur in some samples, while these grade into each other in other samples. Some of the variations are demonstrated in pl. 2, fig. 7 and pl. 3 , fig. 1 .
Lycopodiacidites Couper 1953

Lycopodiacidites rugulatus (Couper) E. Schulz 1967

PI. 3, fig. 3. Assemblage A1, C3; acme A1.

Lycopodiacidites cf. sp. A of Vigran \& Thusu 1975

Assemblage B, C1.

Contignisporites Dettmann 1963

Contignisporites problematicus (Couper) Döring 1965

Pl. 3, fig. 4. Assemblage A1, B, C, D; acmes assemblage $B$ and C3.

Contignisporites dunrobinensis (Couper Schulz 1967

Pl. 3, fig. 5. Assemblage B.

Contignisporites glebulensis Dettmann 1963

P1. 3, fig. 6. Assemblage B.

aff. Contignisporites sp. 1

Assemblage D (sample 41).

Remarks: This species was recorded from the

Ramså Formation of Adøya, Norway as spore species A by Vigran and Thusu, 1975.

aff. Contignisporites sp. 2

Assemblage C1, (C2).

Remarks: This species was probably recorded by Vigran \& Thusu (1975) as Corrugatisporites sp. cf. C. amplectiformis from the Hestberget Member of Ramså Formation. However, the sculptural elements are higher (up to $5 \mu \mathrm{m}$ ), in the Greenland specimen.

Klukisporites Couper 1958

Klukisporites variegatus Couper 1958

P1. 3, fig. 7. Assemblage B, C3, D (samples 49-

51 and 58); acme assemblage $B$

Klukisporites pseudoreticulatus Couper 1958

Pl. 3, fig. 8. Assemblage C3, D lower part.

Klukisporites sp. 1.

Pl. 3, fig. 9. Assemblage D.

Staplinisporites Pocock 1962

Staplinisporites caminus (Balme) Pocock 1962

Pl. 4, fig. 1. Assemblage (C), C1, C2, D lower part; acme (C).

Sestrosporites Dettmann 1963

Sestrosporites pseudoalveolatus (Couper) Dettmann 1963

Pl. 4, fig. 2. Assemblage C1, D lower part.

Subturma: Zonotriletes Waltz

Neochomotriletes Reinhardt 1961

Neochomotriletes triangularis (Bolkhovitina)

Reinhardt 1961 

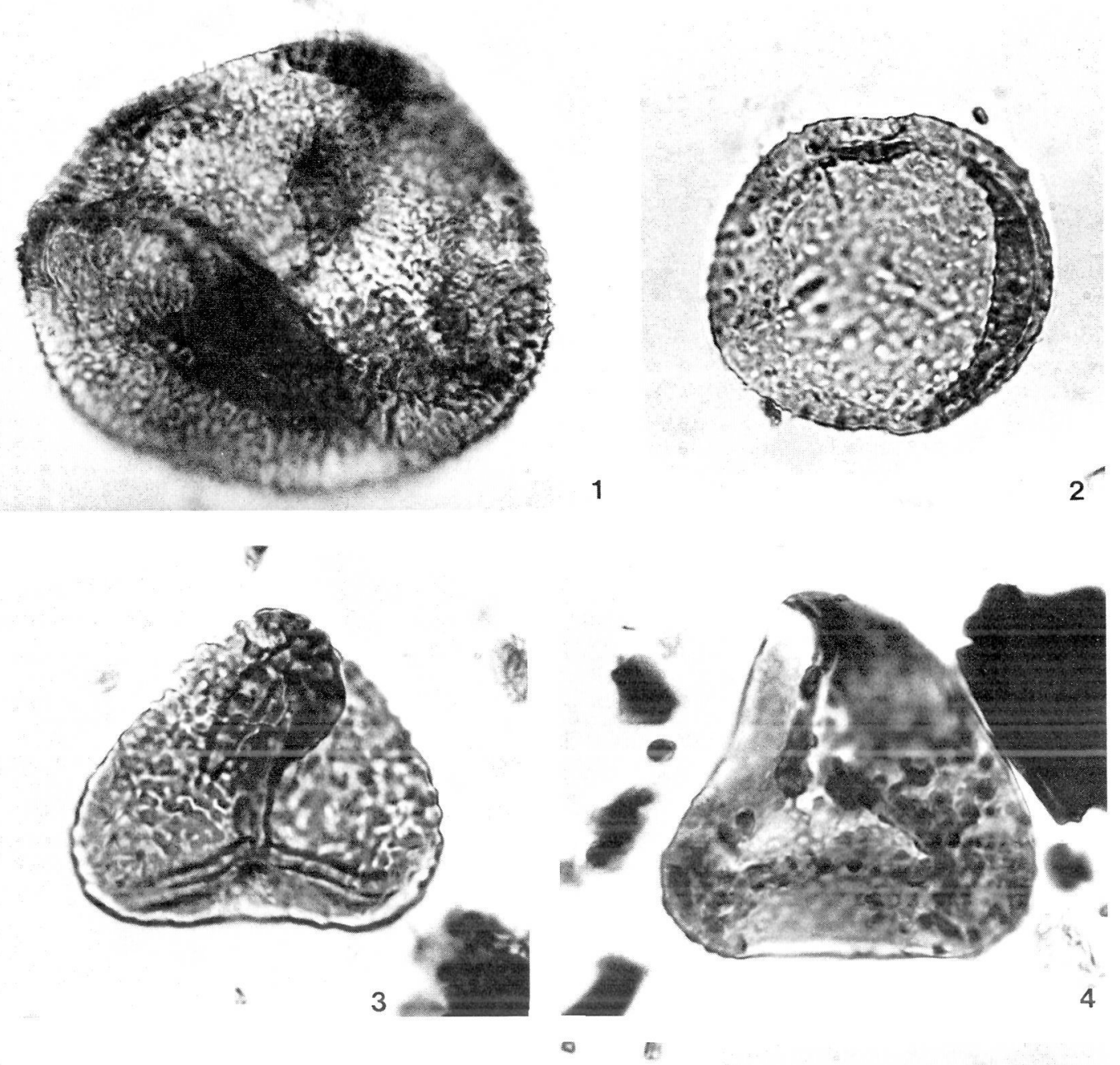

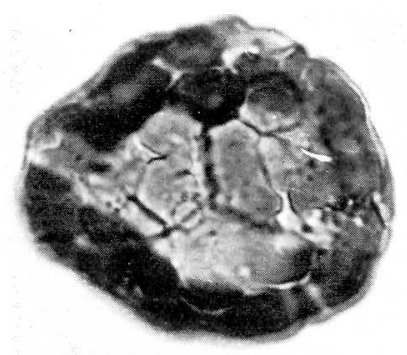

$2 \cos ^{2}$

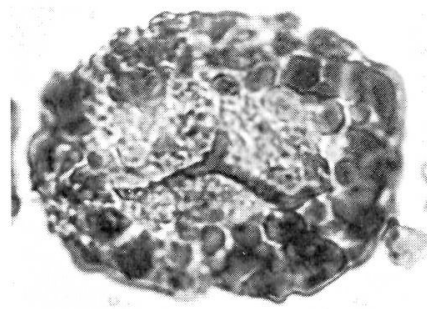

3

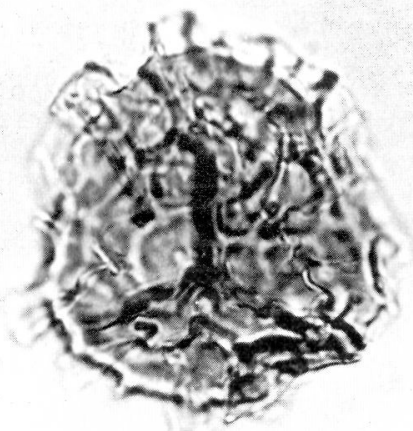

Plate 2. Fig. 1; aff. Baculatisporites sp. 3. Neill Klinter F., (142823), 3-1). MGUH 16595. Fig. 2; Apiculatisporis ovalis (Nilsson) Norris. Neill Klinter F., (142821, 3-1). MGUH 16596. Fig. 3; Trachysporites? fuscus Nilsson. Hareelv F., (142851, 3-3). MGUH 16597. Fig. 4; Manumia cf. irregularis Pocock Vardekløft F., (142838, 4-1). MGUH 16598. Fig. 5; Leptolepidites major Couper. Vardekløft F., (142834, 4-5). MGUH 16599. Fig. 6; Leptolepidites bossus (Couper) E. Schulz. Vardekløft F., (142835, 4-2). MGUH 16600. Fig. 7; Lycopodiumsporites sp. Vardekløft F., (142832, 4-6). MGUH 16601. 
Assemblage C1.

Densoisporites (Weyland \& Krieger) Dettmann 1963

Densoisporites velatus Weyland \& Krieger 1953

Assemblage C2, D.

Heliosporites E. Schulz 1962

Heliosporites altmarkensis E. Schulz 1962

Pl. 4, fig. 3. Assemblage A2.

cf. Heliosporites sp.

Assemblage C3.

Remarks: Size $60 \mu \mathrm{m}$, zona reduced.

Perotriletes Couper 1953

Perotriletes sp.

Assemblage D (sample 58).

Turma: Monoletes Ibrahim

Marattisporites Couper 1958

Marattisporites scabratus Couper 1958

Assemblage B, C2, C3, D middle part.

Anteturma: Pollenites R. Potonié

Turma: Saccites Erdtman

Subturma: Disaccites Cookson

Vitreisporites Leschik 1955

Vitreisporites pallidus (Reissinger) Nilsson 1958

Pl. 4, fig. 4. Assemblage C, D; rare in A1.

Pinuspollenites (Raatz) Potonié 1956

Pinuspollenites pinoides (Nilsson) Lund 1977

P1. 4, fig. 5. Assemblage A, D middle and upper part.

Pinuspollenites minimus (Couper) Kemp 1970

Assemblage A, B, C1, C3, D.

Podocarpidites Cookson ex Couper 1953

Podocarpidites rousei Pocock 1970

Pl. 4, fig. 6. Assemblage C3, D lower (and middle) part.

Podocarpidites cf. langii Pocock 1970

Assemblage A1, C3.

Remarks: Similar to $P$. rousei, but with less folded and more laterally orientated sacci.

Protopinus Bolkhovitina 1956

Protopinus scanicus Nilsson 1958

Assemblage A, D; acme A1.

Alisporites (Daugherty) Jansonius 1971

Alisporites microsaccus (Couper) Pocock 1962

Pl. 4, fig. 8. Assemblage D.

Quadraeculina Maljavkina 1949

Quadraeculina anellaeformis Maljavkina 1949

P1. 4, fig. 7. Assemblage B, C1, C2.

Undifferentiated disaccate pollen

Assemblage A, B, C, D.
Remarks: This group comprises mainly species of Alisporites and Abietineapollenites. They are common in all assemblages except A2 and B.

Turma: Aletes Ibrahim

Subturma: Azonaletes (Luber) Potonié \& Kremp Araucariacites Cookson 1947

Araucariacites australis Cookson 1947

Assemblage A1, B, C, D.

Perinopollenites Couper 1958

Perinopollenites elatoides Couper 1958

PI. 5, fig. 3. Assemblage A, B, C, D; acme C1.

Cypraessacites (Bolkhovitina 1958) Krutzsch 1971

Cypraessacites spp.

Assemblage A, B, C, D.

Cerebropollenites Nilsson 1958

Cerebropollenites macroverrucosus (Thiergart)

E. Schulz 1967

Pl. 5, fig. 1. Assemblage A, B, C, D; distinctively more common in $\mathrm{C}$ and $\mathrm{D}$ than in $\mathrm{A}$ and $\mathrm{B}$.

Cerebropollenites thiergartii E. Schulz 1967

Pl. 5, fig. 2. Assemblage A, B, C, D; acme in assemblage A.

Spheripollenites Couper 1958

Spheripollenites subgranulatus Couper 1958

Pl. 5, fig. 4. Assemblage A, B, C, D; dominant in assemblage $\mathrm{B}$.

Exesipollenites Balme 1957

Exesipollenites tumulus Balme 1957

Pl. 5, fig. 5. Assemblage D middle part (sample 51) and higher.

Subturma: Zonaletes

Callialasporites Dev 1961

Callialasporites dampieri (Balme) Dev 1961

Pl. 5, fig. 6. Assemblage C, D; acmes C3 and D upper part.

Callialasporites trilobatus (Balme) Dev 1961

Assemblage C3, D middle part.

Callialasporites turbatus (Balme) E. Schulz 1967

Pl. 5, fig. 7. Assemblage C2, C3, D upper part.

cf. Callialasporites sp.

Pl. 6, fig. 1 Assemblage C1, C2, D upper part.

Remarks: This form is intermediate between

Callialasporites and Araucariacites.

Turma: Plicates (Naumova) R. Potonié

Subturma: Monocolpates Iversen \& Troels-Smith Chasmatosporites Nilsson 1958 

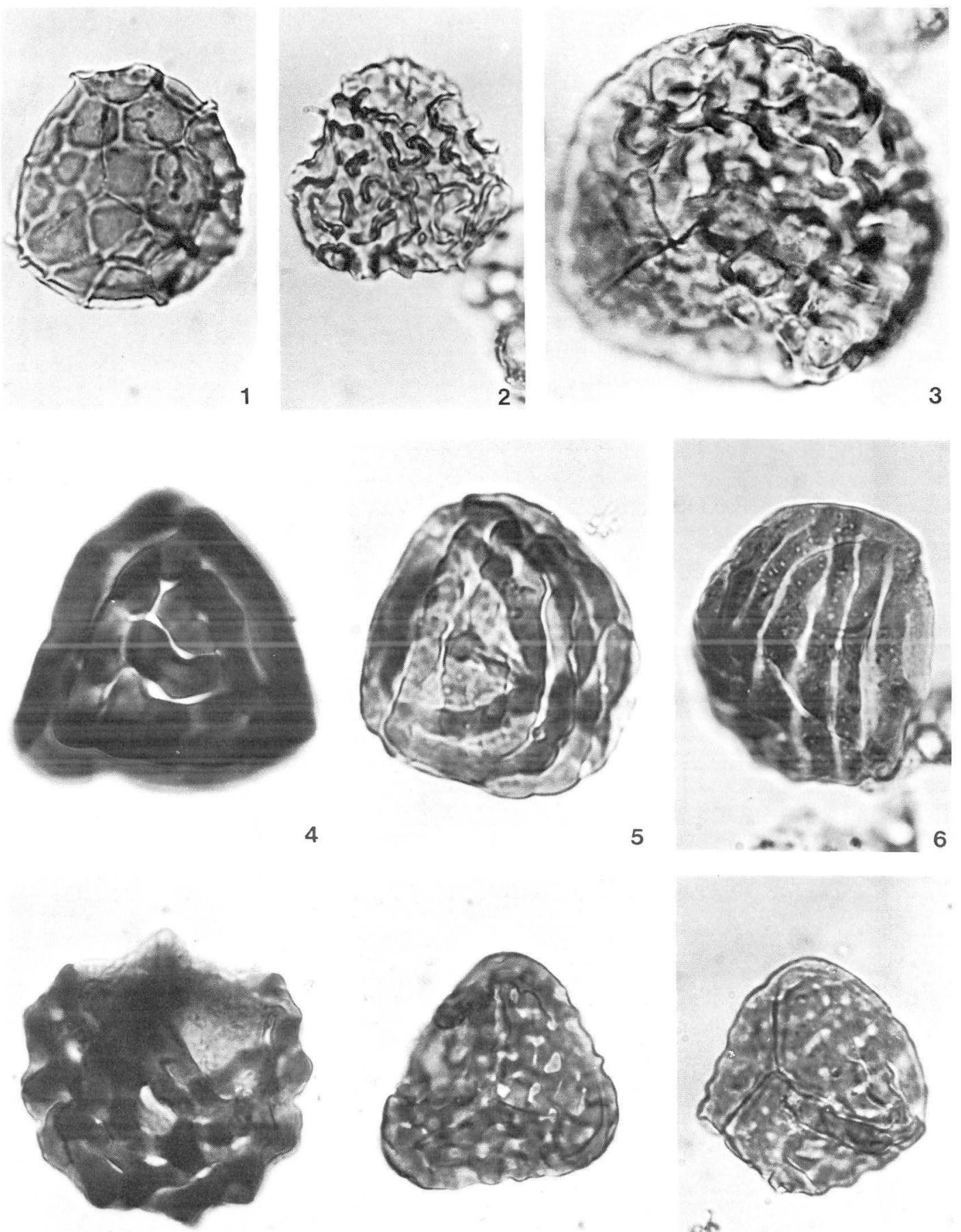

8<smiles>[C]1C2=CC1C2</smiles>

Plate 3. Fig. 1; Lycopodiumsporites sp. Vardekløft F., (142833, 4-4). MGUH 16602. Fig. 2; Lycopodiumsporites semimuris DanzéCorsin \& Laveine. Vardekløft F., (142840, 9-8). MGUH 16603. Fig. 3; Lycopodiacidites rugulatus (Couper) E. Schulz. Vardekløft F., (142840, 9-6). MGUH 16604. Fig. 4; Contignisporites problematicus (Couper) Döring. Vardekløft F., (142839, 6-2. MGUH 16605. Fig. 5; Contignisporites dunrobinensis (Couper) E. Schulz. Neill Klinter F., (142828, 4-4). MGUH 16606. Fig. 6; Contignisporites glebulensis Dettmann. Neill Klinter F., (142828, 4-7). MGUH 16607. Fig. 7; Klukisporites variegatus Couper. Neill Klinter F., (142829, 4-3). MGUH 16608. Fig. 8; Klukisporites pseudoreticulatus Couper. Vardekløft F., (142839, 6-2). MGUH 16609. Fig. 9; Klukisporites sp. 1. Hareelv F., (142851, 3-3). MGUH 16610. 
Chasmatosporites hians Nilsson 1958/C. elegans Nilsson 1958

P1. 6, fig. 2. Assemblage A1, B, C1, C3, D middle part; acme A1.

Chasmatosporites apertus (Rogalska) Nilsson 1958

Assemblage A1, B.

Monosulcites Cookson 1947

Monosulcites minimus Cookson 1947

P1. 6, fig. 3. Assemblage A, C1, C2, D; acme in assemblage $\mathrm{D}$ (sample 51 and higher).

Subturma: Praecolpates Potonié \& Kremp

Eucommiidites (Erdtman) Couper 1958

Eucommiidites troedssonii Erdtman 1948

Assemblage A1, C1, D middle part.

Turma: ? Poroses (Naumova) Potonié

Subturma: ? Monoporines Naumova

Corollina (Maljavkina) Cornet \& Traverse 1975

Corollina torosus (Reissinger) Klaus 1960

Assemblage A1, B, C, D; rare in assemblage D.

Corollina sp. 1

Pl. 6, fig. 4. Assemblage C, D upper part; acme $\mathrm{C} 1, \mathrm{C} 2$.

Remarks: Corollina sp. 1 differs from C. torosus by having continuous endexinal ribs at the equator.

Corollina sp. 2

Pl. 6. fig. 5. Assemblage D (sample 51 only).

Remarks: This species is characterized by a scabrate (microechinate) sculpture. It appears to be related to the spined Corollina/Classopollis species which have their maximum development in the topmost Jurassic.

\section{Dinoflagellate cysts}

Dinoflagellate cysts with apical archaeophyles Adnatosphaeridium Williams \& Downie in Davey et al. 1966

Adnatosphaeridium aemulum (Deflandre) Williams \& Downie 1969

Pl. 7, fig. 1. Assemblage D lower to middle part (samples 43-49).

Remarks: Below (1982) provided sound arguments against the generally used combination of the genus Adnatosphaeridium of which the Tertiary typespecies is lenticular shaped, and the more spherical Jurassic species aemulum. Here a conventional approach is followed.
Ambonosphaera Fensome 1979

Ambonospahera sp.

Assemblage D middle part (sample 47).

Atopodinium Drugg 1978

Atopodinium prostatum Drugg 1978

Pl. 7, fig. 2. Assemblage D middle part (sample

47).

Cassiculosphaeridia Davey 1969

Cassiculosphaeridia dictydia (Sarjeant) Riley \&

Fenton 1982

Assemblage D middle part (sample 47).

Cassiculosphaeridia cf. magna Davey 1969

Assemblage D ?lower to middle part (samples ?43-51)

Cassiculosphaeridia sp.

Pl. 7, fig. 4. Assemblage D lower to middle part (samples 43-50).

Cleistosphaeridium Davey 1966

Cleistosphaeridium sp.

Assemblage D lower part (sample 43).

Remarks: The wall between the spines is irregularly microreticulate.

Compositosphaeridium Dodekova 1974

Compositosphaeridium costatum (Davey \& Williams) Dodekova 1974

PI. 7, fig. 3. Assemblage D middle part (sample 49).

Meiourogonyaulax Sarjeant in Davey et al. 1966

Meiourogonyaulax sp.

Pl. 7, fig. 5. Assemblage D middle part (sample 47 frequent).

Remarks: Contrary to the figured specimen, most specimens lack an operculum. This species was recorded by Piasecki (1980) from the Middle Oxfordian tenuiserratum Zone.

Polystephanephorus Sarjeant 1961

Polystephanephorus cf. paracalathus (Sarjeant) Downie \& Sarjeant 1964

Assemblage D lower to middle part (samples 4147).

Remarks: This "species" can be described as an Adnatosphaeridium aemulum, in which the single processes are dissolved into penitabular process groups. It differs from the holotype (Sarjeant 1976, pl. 6, fig. 1-2) by the presence of small spines on the trabeculae.

Prolixosphaeridium (Davey) Davey 1969

Prolixosphaeridium sp.

Pl. 7, fig. 7. Assemblage D lower part (samples 41-43). 

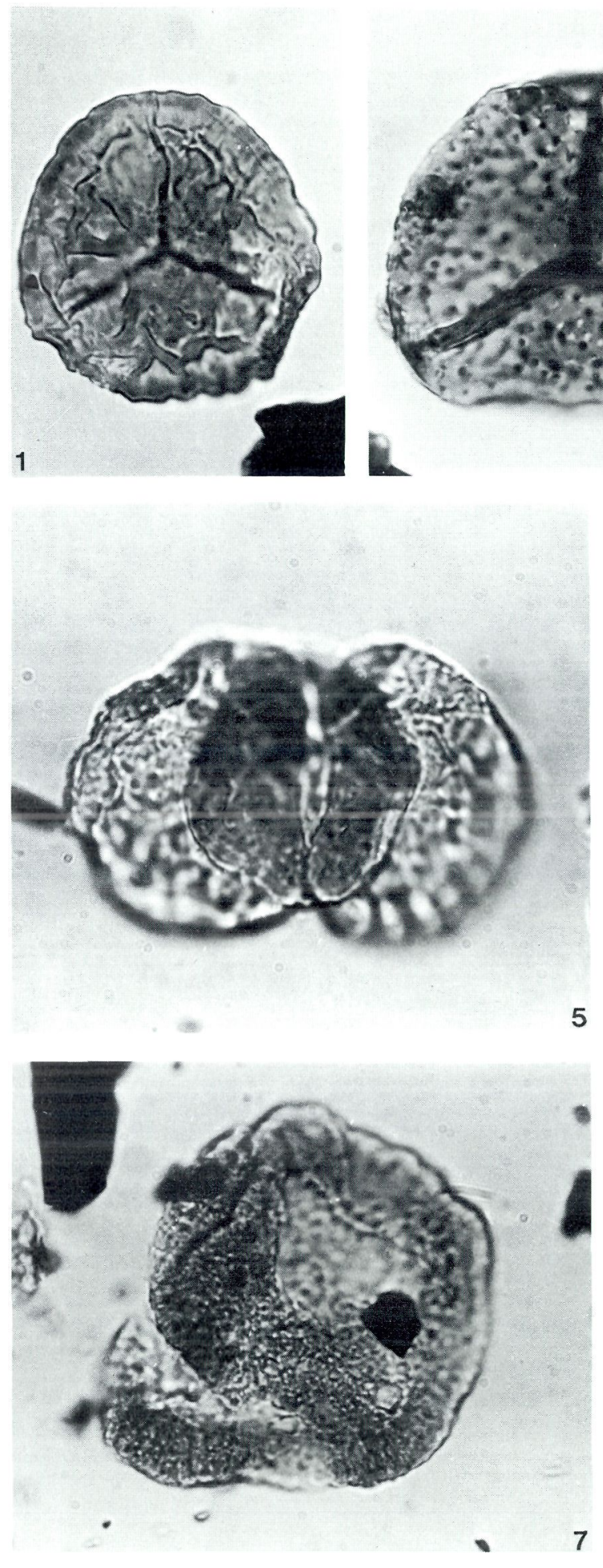

5
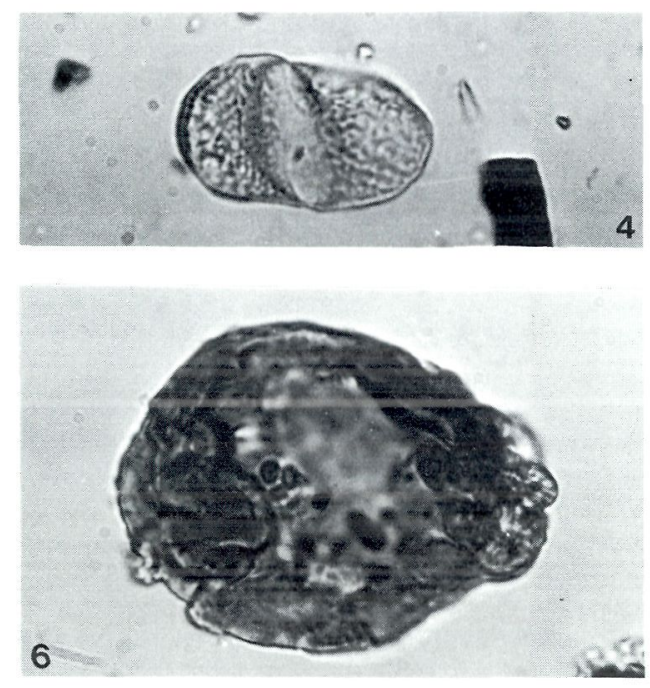

7

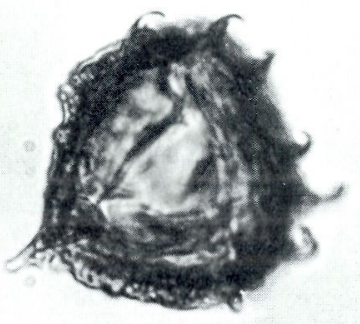

3

Plate 4. Fig. 1; Staplinisporites caminus (Balme) Pocock. Vardekløft F., (142835, 4 4). MGUH 16611. Fig. 2; Sestrosporites pseudoalveolatus (Couper) Dettmann. Hareelv F., (142844, 3-1). MGUH 16612. Fig. 3; Heliosporites altmarkensis E. Schulz. Neill Klinter F., (142827, 4-2). MGUH 16613. Fig. 4; Vitreisporites pallidus (Reissinger) Nilsson. Vardeelv F., (142832, 4-2). MGUH 16614. Fig. 5; Pinuspollenites pinoides (Nilsson) Lund. Neill Klinter F., (142827, 4-1). MGUH 16615. Fig. 6; Podocarpidites rousei Pocock. Hareelv F., (142844, 3-2). MGUH 16616. Fig. 7; Quadraeculina anellaeformis Maljavkina. Vardeelv F., (142836, 4-3). MGUH 16617. Fig. 8; Alisporites microsaccus (Couper) Pocock. Hareelv F., (142858, 3-1). MGUH 16618. 
Remarks: This species is similar to $P$. mixtispinosum (Klement) Davey et al., 1969, but is between the larger spines irregularly microreticulate rather than spined.

Sentusidinium Sarjeant \& Stover 1978

Sentusidinium pelionense Fensome 1979

PI. 7, fig. 8. Assemblage C; acme samples 32-34 $(-36)$.

Sentusidinium rioultii (Sarjeant) Sarjeant \& Stover 1978

Assemblage D lower part (samples 43-44).

Sentusidinium sp. 1 of Piasecki 1980

Pl. 7. fig. 6. Assemblage D lower to middle part (sample 41-49).

Remarks: This species resembles $S$. sparsibarbatum Erkmen \& Sarjeant 1980, but differs in having straight sides. It was described by Piasecki (1980) from the Early Oxfordian (cordatum Z.) to Late Oxfordian (rosenkrantzii Z.)

Stephanelytron (Sarjeant) Storer et al. 1977

Stephanelytron redcliffense Sarjeant 1961

PI. 7, fig. 9. Assemblage D middle part (sample 49).

Systematophora Klement 1960

Systematophora aff. areolata Klement 1960

Assemblage D upper part (sample 57).

cf. Systematophora sp.

PI. 7. fig. 10 Assemblage D upper part (samples 55-57).

Remarks: The penitabular process groups tend to have a membrane at their bases. Some process tips are broadly forked indicating very incomplete ring trabeculae. An archaeopyle was not observed.

Dinoflagellate cysts with intercalary archaeopyles.

Kalyptea Cookson \& Eisenack 1960 sensu lato Kalyptea cf. glabra (Cookson \& Eisenack) Wiggins 1975

Pl. 7, fig. 11. Assemblage D middle part (sample 51).

Remarks: The apical horn is considerably longer in the Greenland forms than in the Australian type material. This species was found in the Middle Oxfordian, densiplicatum Z. - basal glosense $Z$. by Piasecki (1980) and referred to $K$. diceras Cookson \& Eisenack 1960, which, however, is more spindelshaped and possesses a distinct kalyptra. Possibly our specimens should be placed in a new subspecies of $K$. glabra or in a new spe- cies. However, it is too rare in the material examined to justify the erection of a new taxon. Kalyptea jurassica Alberti 1961

Assemblage D middle part (sample 49).

cf. Kalyptea monoceras Cookson \& Eisenack 1960

Pl. 7, fig. 12. Assemblage D middle part (samples 47-49).

Remarks: The apical horn is shorter and the "kalyptra lower than in the type material. Moreover the "kalyptra" shows linear structures reminiscent of sutural ridges.

Paragonyaulacysta Johnson \& Hills 1973

Paragonyaulacysta borealis (Brideaux \& Fisher) Stover \& Evitt 1978

Assemblage D upper part (sample 57).

Parvocysta Bjærke 1980

Parvocysta contracta Bjærke 1980a

Assemblage C1 (sample 31).

Pareodinia Deflandre 1947

Pareodinia ceratophora Deflandre 1947

Assemblage C3, D upper part (samples 39-57).

Pareodinia sp.

Assemblage D middle part (sample 47).

Remarks: Characterized by its smooth wall, small apical horn, and large size (ca. $95 \times 70$ $\mu \mathrm{m})$.

Undetermined taxa.

Single specimens of three new taxa with intercalary archeopyles were observed in the Neil Klinter Formation. In assemblage A (sample 20) a triangular ?Phallocysta and in assemblage B a broad Diconodinium like form (sample 29) were observed. An acavate genus with two antapical horns was noted in sample 28 .

Dinoflagellate cysts with precingular archeopyles.

Acanthaulax Sarjeant 1968

Acanthaulax areolata (Sarjeant) Riley \& Fenton 1982

PI. 8. fig. 3. Assemblage D lower part (sample 41-44).

Acanthaulax sp. .

Assemblage D middle part (sample 49).

Aldorfia Stover \& Evitt 1978

cf. Aldorfia sp.

Assemblage D middle part (sample 47, frequent).

Remarks: The archaeopyle could not be determined. 

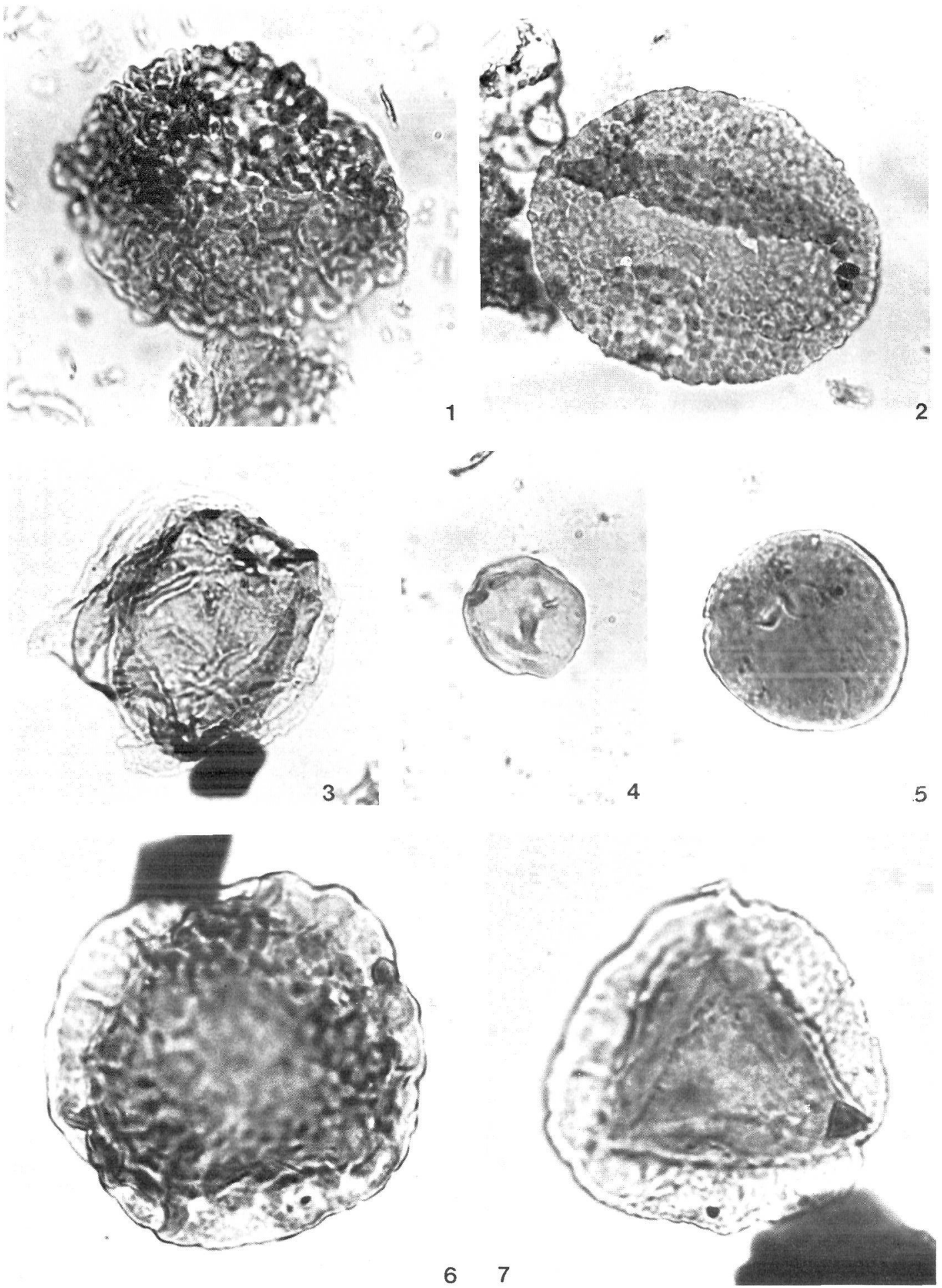

Plate 5. Fig. 1; Cerebropollenites macroverrucosus (Thiergart) E. Schulz). Neill Klinter F., (142828, 4-2). MGUH 16619. Fig. 2; Cerebropollenites thiergartii E. Schulz. Hareelv F., (142858, 3-1). MGUH 16620. Fig. 3; Perinopollenites elatoides Couper. Neil Klinter F., (142829, 4-5). MGUH 16621. Fig. 4; Spheripollenites subgranulatus Couper. Neill Klinter F., (142829, 3-1). MGUH 16622. Fig. 5; Exesipollenites tumulus Balme. Hareelv F., (142851, 3-3). MGUH 16623. Fig. 6; Callialasporites dampieri (Balme) Dev. Hareelv F., (142855, 3-2). MGUH 16624. Fig. 7; Callialasporites turbatus (Balme) E. Schulz. Hareelv F., (142858, 3-1). MGUH 16625. 
Chytroeisphaeridia (Sarjeant) Davey 1979

Chytroeisphaeridia chytroeides Sarjeant 1962

Assemblage C3, D middle part (samples 40-49).

Chytroeisphaeridia cerastes Davey 1979

Pl. 7, fig. 13. Assemblage C3, D lower part (samples 40-43).

Gonyaulacysta (Deflandre) Stover \& Evitt 1978

Gonyaulacysta jurassica (Deflandre) Norris \& Sarjeant 1965

Assemblage D (sample ?44-47-58).

Gonyaulacysta jurassica var. longicornis Deflandre 1938

PI. 8, fig. 2. Assemblage D lower-middle part (samples 51-52).

Gonyaulacysta sp.

PI. 8, fig. 1. Assemblage D at top of middle part (samples 51-52).

Remarks: The species differs from $G$. jurassica by the absence of cingulum and in having nearly smooth parasutural crests and a more rounded shape.

Hystrichogonyaulax Sarjeant in Davey et al. 1969

Hystrichogonyaulax cladophora (Deflandre)

Stover \& Evitt 1978

Assemblage D middle to upper part (samples 4758 , partly common).

Leptodinium (Klement) Stover \& Evitt 1978

Leptodinium freakei (Sarjeant) Sarjeant 1969

Assemblage D (sample 45).

Leptodinium sp.

Pl. 8, fig. 4. Assemblage D upper part (samples 55-56).

Remarks: This large species with narrow cingulum is similar to Leptodinium sp. A of Bjærke (1977, pl. 8, figs $7 \&$ 8) from the Oxfordian/Kimmeridgian of Svalbard. However, our specimens differ in having more undulating parasutural crests. A cavate variety occurs in sample 56 and an acavate variety occurs in sample 53.

Scriniodinium Klement 1957

Scriniodinium crystallinum (Deflandre) Klement 1960

Pl. 8, fig. 5. Assemblage D middle to upper part (samples 47-58).

Scriniodinium cf. playfordii Cookson \& Eisenack 1960

Pl. 8, fig. 6. Assemblage D middle part (sample 47-49).

Remarks: The cysts from Greenland differ from the Australian type material in having a less developed apical horn.
Scriniodinium galeritum (Deflandre) Klement 1960

Assemblage C3 to D middle part (samples 4052).

Scriniodinium cf. irregularis (Cookson \& Eisenack) Stover \& Evitt 1978.

Pl. 8, fig. 7. Assemblage D upper part (sample 57).

Remarks: A single specimen was recovered. It differs from the Australian type material in having lower spines.

Tubotuberella Vozzhennikova 1967

Tubotuberella apatela (Cookson \& Eisenack) Ioannides, Stavrinos \& Downie 1977

Assemblage D middle part (sample 47).

Tubotuberella eisenackii (Deflandre) Stover \& Evitt 1978

Assemblage C3, D upper part (samples 40-57).

Tubotuberella sp.

Assemblage D upper part (sample 57).

Remarks: The species is distinguished from $T$. egemenii by the presence of low spines at the parasutural crests.

Dinoflagellate cysts with combination archaeopyles

Carpathodinium Drugg 1978

Carpathodinium predae (Beju) Drugg 1978

Assemblage C3 (sample 40).

Ctenidodinium (Deflandre) Gocht 1970

Ctenidodinium spp.

Assemblage C3 (sample 40).

Remarks: The upper part of the Vardekløft Formation (sample 40) has yielded poorly preserved specimens of the genus Ctenidodinium. Most carry spines, and better preserved specimens would probably be determined as Ctenidodinium stauromatos (Sarjeant) Stover \& Evitt 1978 and possibly also as $C$. ornatum (Eisenack) Deflandre 1938.

Hemicystodinium Wall 1967

cf. Hemicystodinium sp.

Assemblage D (sample 47).

Heteraulacacysta Drugg \& Loeblich 1967

aff. Heteraulacacysta sp.

Assemblage D (samples 45-47).

Luehndea Morgenroth 1970

Luehndea spinosa Morgenroth 1970

Assemblage B (sample 29).

Mancodinium Morgenroth 1970 

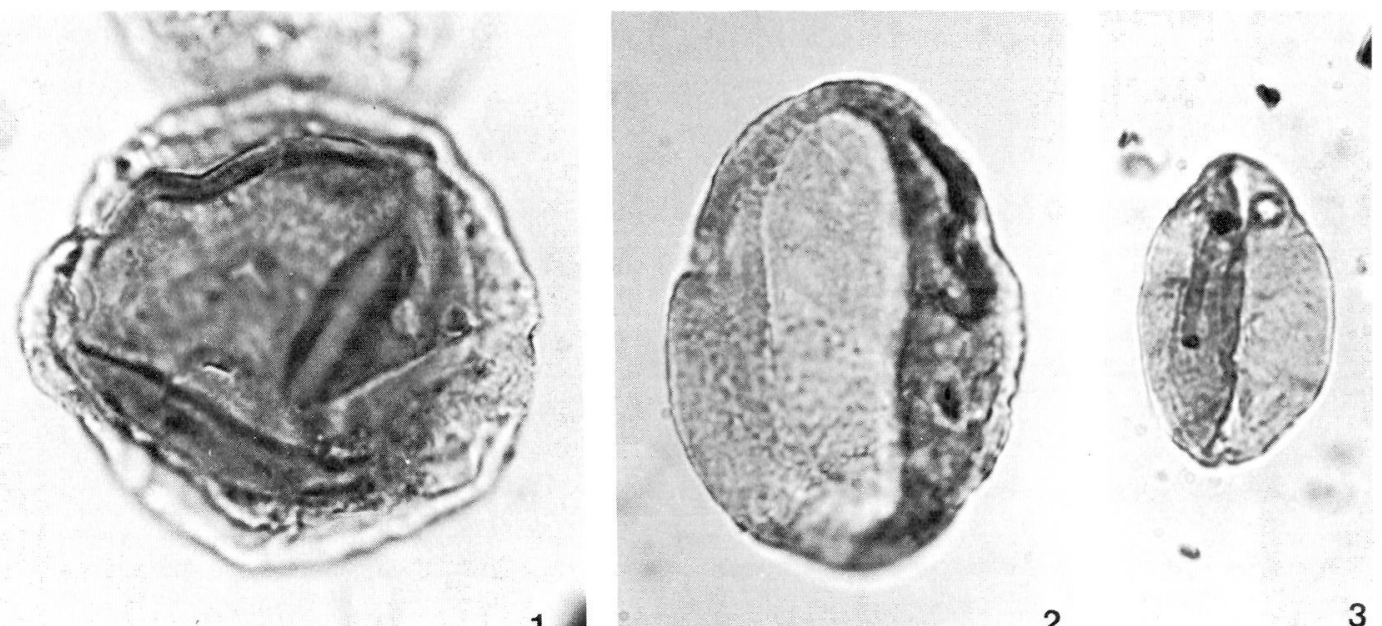

1

2

3

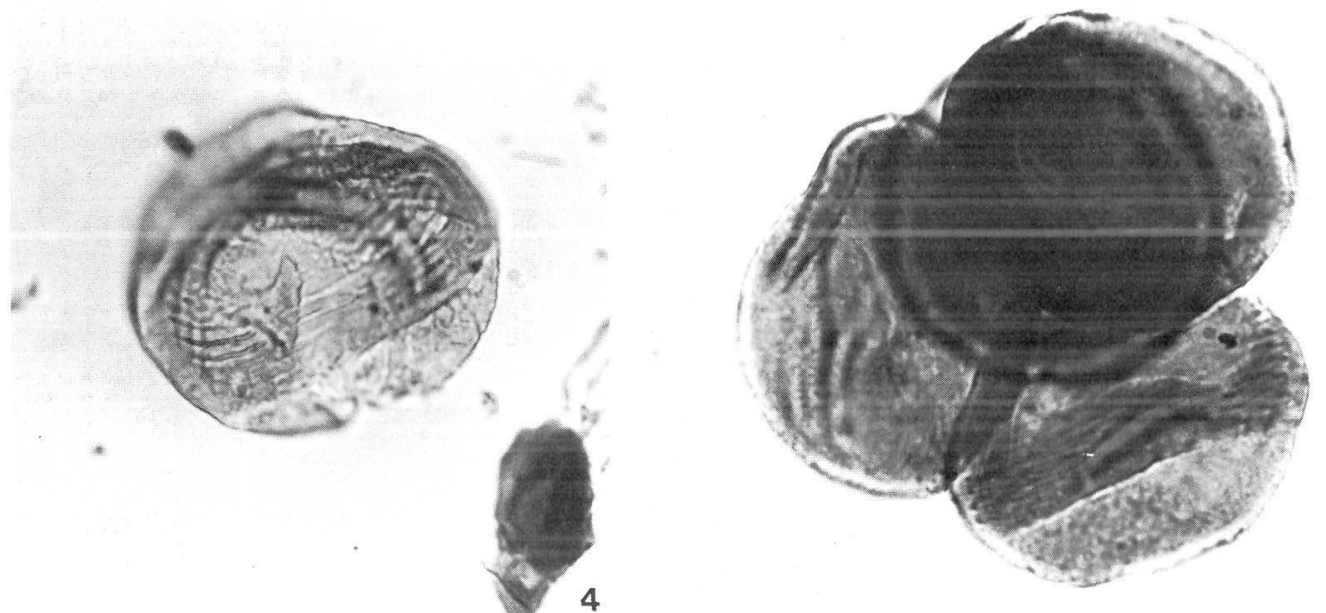

5

Plate 6. Fig. 1; cf. Callialasporites sp. Hareelv F., (142858, 3-3). MGUH 16626. Fig. 2; Chasmatosporites hians Nilsson/C. elegans Nilsson. Neill Klinter F., (142821, 4-7). MGUH 16627. Fig. 3; Monosulcites minimus Cookson. Hareelv F., (142851, 3-3). MGUH 16628. Fig. 4. Corollina sp. 1. Vardekløft F., (142833, 3-1). MGUH 16629. Fig. 5; Corollina sp. 2. Hareelv F., (142851, 3-3). MGUH 16630 .

Mancodinium semitabulatum Morgenroth 1970

Pl. 8, fig. 8. Assemblage B (sample 29).

Mancodinium sp.

Pl. 8, fig. 9. Assemblage B (sample 29).

Nannoceratopsis (Deflandre) Evitt 1961

Nannoceratopsis gracilis Alberti 1961

Assemblage B, C1 (sample 29, 31, rare).

Nannoceratopsis cf. plegas Drugg 1978

Assemblage C3 (sample 40).

Nannoceratopsis senex van Helden 1977

Pl. 8, fig. 10. Assemblage B (samples 28-29, abundant).
Nannoceratopsis triceras Durgg 1978

Assemblage A1 (sample 21).

Remarks: The determination of the specimen is uncertain due to the poor preservation.

Sirmiodinium Alberti 1961

Sirmiodinium grossii Alberti 1961

Assemblage D (samples 41-58, common in 41 and 43).

Sirmiodinium sp.

Assemblage D middle part (sample 47).

Wanaea Cookson \& Eisenack 1958

Wanaea acollaris Dodekova 1975 
Assemblage D middle part (sample 49).

Remarks: The species has previously been recovered only from Callovian and older strata (Riley \& Fenton 1982) and may be reworked.

? Wanaea sp.

Assemblage D middle part (sample 47).

Remarks: A single fragmented semi-conical hypocyst with parasutural ridges and reticulate sculpture was found.

This distinctive form could also be assigned to ?Ctenidodinium. In the North Sea this species occurs slightly above the level characterized by typical Wanaea (R. Dunay, personal communication).

\section{Other algae}

Veryhachium (Deunff) Downie \& Sarjeant 1963 Veryhachium sortehatense Fensome 1979

Assemblage A1; frequent in sample 21.

Veryhachium spp.

Assemblage A1, D middle part (sample 46).

Micrhystridium Deflandre 1937

Micrhystridium spp.

Assemblage A1, B, C1, C3.

Tasmanids

Assemblage A2, B, C3, D middle and upper part.

Remarks: Only few tasmanids (Tasmanites Newton/Crassosphaera Cookson \& Manum 1960 and Campenia Mädler 1968) were recovered from the studied sequence of Jameson Land.

\section{Palynostratigraphy}

Spores, pollen and dinoflagellate cysts are well represented in the samples studied. Special emphasis is placed on spores and pollen for which a sequence of assemblages (A-D) is established (fig. 4,5 ). The taxa used in defining the assem- blages are sufficiently widely distributed to be recognized over a wider geographical area including parts of NW Europe. The associated dinoflagellate cysts are mainly used to improve the age correlation of the mentioned spore-pollen assemblages. When detailed data on dinoflagellate cyst distribution in more sections from the area are published, it will be possible to propose a precise dinoflagellate zonation for the Late Jurassic of East Greenland.

\section{Spore-pollen assemblages A-D}

The microfloral assemblages A-D differ from those of the underlying Kap Stewart Formation (Pedersen \& Lund, 1980) by the presence of Cerebropollenites macroverrucosus and the absence of such older taxa as Ricciisporites tuberculatus.

Assemblage A (samples 20-27) is characterized by aff. Baculatisporites sp. 3 and the acme of Cerebropollenites thiergartii. Subassemblage A1 (samples 20-23) is well distinguished by acmes of aff. Baculatisporites sp. 3, Baculatisporites sp. 2, Lycopodiacidites rugulatus, and Apiculatisporis ovalis. Subassemblage A2 (samples 26-27) is defined by the absence of the characteristic A1 and B taxa (acme of Baculatisporites sp. 3 etc., very common Spheripollenites). Heliosporites altmarkensis and Iraquispora laevigata were observed only in A2. However, they have been recovered from the underlying Kap Stewart Formation as well (Pedersen \& Lund, 1980).

Assemblage B (sample 28-29): the most characteristic feature of this assemblage is the mass occurrence of Spheripollenites subgranulatus. This species comprises nearly $75 \%$ of all palynomorph specimens in sample 28. The incoming of Klukisporites variegatus, Leptolepidites bossus, Manumia cf. irregularis, as well as species of Contignisporites (C. dunrobinens, C. glebulens) and acme of $C$. problematicus are also diagnostic.

Plate 7. Fig. 1; Adnatosphaeridium aemulum (Deflandre) Williams \& Downie. Hareelv F., (142844, 3-1). MGUH 16631. Fig. 2; Atopodinium prostatum Drugg. Hareelv F., (142847, 8-4). MGUH 16632. Fig. 3. Compositosphaeridium costatum (Davey \& Williams) Dodekova. Hareelv F., (142849, 3-1). MGUH 16633. Fig. 4; Cassiculosphaeridia sp. Hareelv F., (142843, 13-1). MGUH 16634. Fig. 5; Meiourogonyaulax sp. Hareelv F., (142847, 3-1). MGUH 16635. Fig. 6; Sentusidinium sp. 1 of Piasecki. Hareelv F., (142847, 5-5). MGUH 16636. Fig. 7; Prolixosphaeridium sp. Hareelv F., (142843, 11-1). MGUH 16637. Fig. 8; Sentusidinium pelionense Fensome. Vardekløft F., (142832, 4-6). MGUH 16638. Fig. 9; Stephanelytron redcliffense Sarjeant. Hareelv F., (142849, 3-2). MGUH 16639. Fig. 10; cf. Systematophora sp. Hareelv F., (142855, 3-1). MGUH 16640. Fig. 11; Kalyptea cf. glabra (Cookson \& Eisenack) Wiggins. Hareelv F., (142851, 3-2b). MGUH 16641. Fig. 12; cf. Kalyptea monoceras Cookson \& Eisenack. Hareelv F., (142847, 5-6). MGUH 16642. Fig. 13; Chytroeisphaeridia cerastes Davey. Vardekløft F., (142840, 9-6). MGUH 16643. 

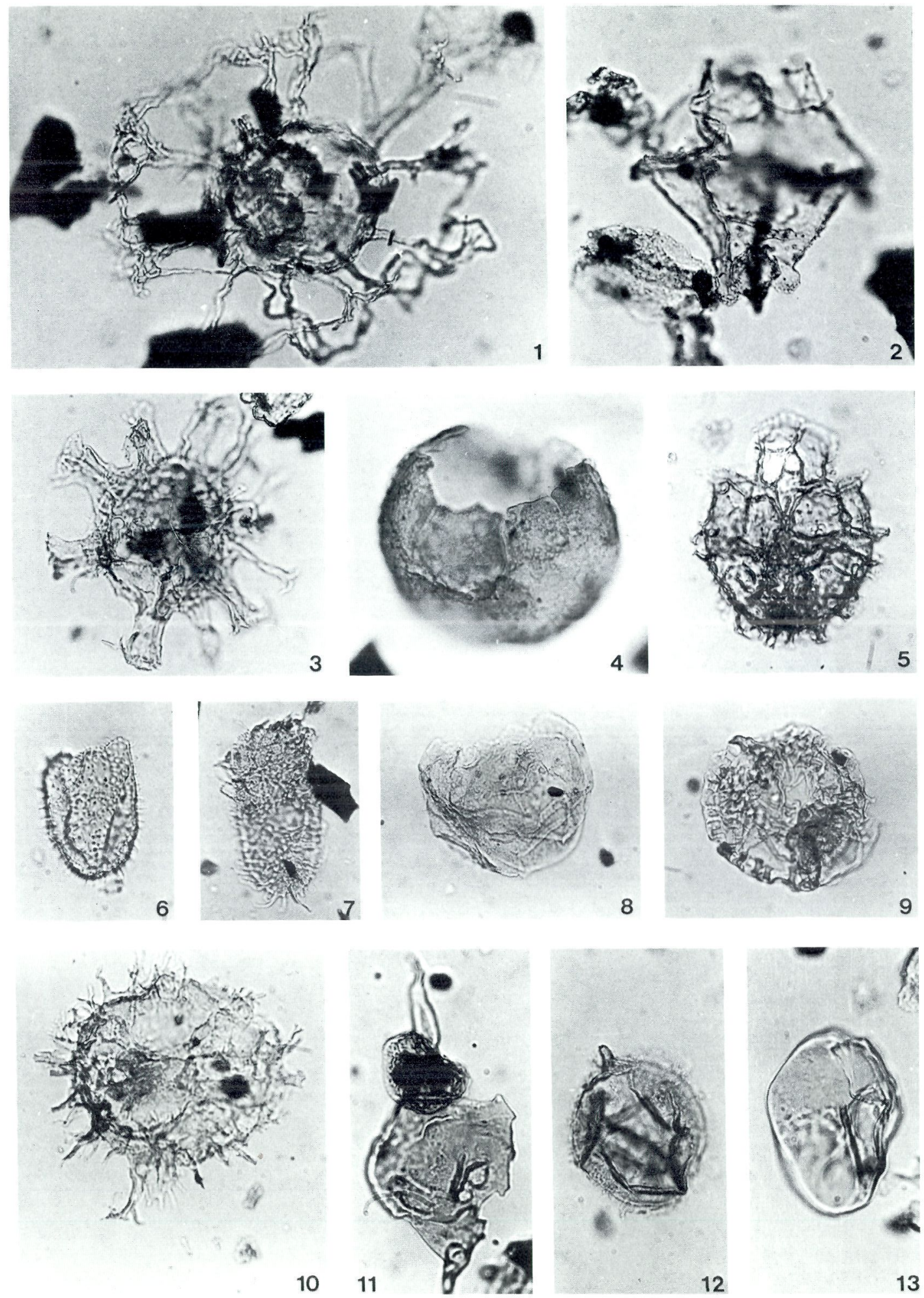

9

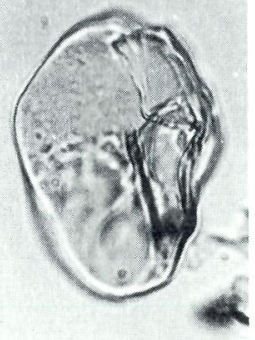


Assemblage C (sample ?31, 32-40): the base is defined by the appearance of genera Callialasporites, Sestrosporites and Staplinisporites. The top is defined by the incoming of taxa in the overlaying assemblage. The top also coincides with the highest observed occurrence of Lycopodiacidites rugulatus, Contignisporites problematicus and of frequent Baculatisporites. Leptolepidites major is apparently restricted to assemblage $\mathrm{C}$ and the acme of Corollina sp. occurs here. Cerebropollenites macroverrucosus is much more common in assemblage $\mathrm{C}$ and $\mathrm{D}$ than in underlaying assemblages. Subassemblage $\mathrm{C} 1$ (sample 33-35) shows a maximum of Perinopollenites elatoides. In subassemblage C2 (samples 36-38) Callialasporites turbatus and Densoisporites velatus appear. Quadraeculina anellaeformis has its acme in C1-C2 and was not observed higher. Subassemblage C3 (samples 39-40 is characterized by the appearance of Klukisporites pseudoreticulatus and Podocarpidites rousei. Compared with the previously known ranges of the characteristic taxa, the distinction between $\mathrm{C} 2$ and $\mathrm{C} 3$ is probably of wider areal use.

Sample 31, collected at $425 \mathrm{~m}$ in the upper part of the Neill Klinter Formation is only referred to as (C) because, of all the above mentioned characteristics of Assemblage C, only Staplinisporites occur. The Vardekløft Formation is thus distinguished from the Neill Klinter Formation by the presence of Callialasporites and higher numbers of Cerebropollenites macroverrucosus.

Assemblage D (samples 41-58) is characterized by the incoming of Gleicheniidites, especially of the slightly pitted $G$. conspiciendus. The large Deltoidospora sp. 1 and Klukisporites sp. 1 are also charateristic. Monosulcites minimus is frequent from sample 51 upwards. However, this is considered to be a local characteristic and thus not usable for establishing a subassemblage. The dinoflagellate cysts show a clear succession in assemblage $\mathrm{D}$, as described below.

\section{Dating of assemblage A-D}

The dinoflagellate cysts are subordinate in number to pollen and spores (fig. 7), but they provide important evidence for stratigraphical correlations. The occurrence of selected dinoflagellate cysts is shown in figure 6 .
Subassemblage A1 (Neill Klinter Formation, lower part)

The macrofauna dates this section as Pliensbachian (Surlyk et al., 1973). The microfloral datings are less firm although the maximum occurrence of Baculatisporites spp. is consistent with an Early Pliensbachian age, when compared with NW Europe (Morbey, 1978). The presence of Nannoceratopsis triceras/"Paranannoceratopsis triradis" tentatively exclude an age older than the Late Pliensbachian (Drugg, 1978; Morbey, 1978). The subassemblage A1 microflora is thus of an Early Jurassic, Pliensbachian, probably Late Pliesbachian age.

Subassemblage A2 (Neill Klinter Formation, middle part)

The presence of Heliosporites altmarkensis (Krauselisporites reissingerii) points to an age not younger than the Late Pliensbachian (Morbey, 1978). Considering the (Late) Pliensbachian age of the underlying and the superjacent intervals, a Late Pliensbachian age is also probable for subassemblage A2.

Assemblage B (Neill Klinter Formation, middle part)

The dinoflagellate cysts Nannoceratopsis senex, Mancodinium semitabulatum, and especially Luehndea spinosa indicate a Late Pliensbachian to Early Toarcian age (Wille, 1982; Wollam \& Riding, 1983). A distinct maximum of Spheripollenites indicates an Early Toarcian age (Morbey, 1978). An Early Jurassic, Early Toarcian (to Late Pliensbachian) age is thus probable for assemblage $\mathrm{B}$.

"Subassemblage" (C) (Neill Klinter Formation, upper part)

The presence of the dinoflagellate cyst Parvocysta contracta known hitherto only from the Toarcian of Spitsbergen (Bjærke, 1980a) and of the spore Staplinisporites telatus, which is well known from Late Toarcian and younger floras (Schulz, 1967) suggests a Lower Jurassic, (Late) Toarcian age for "subassemblage" (C). 

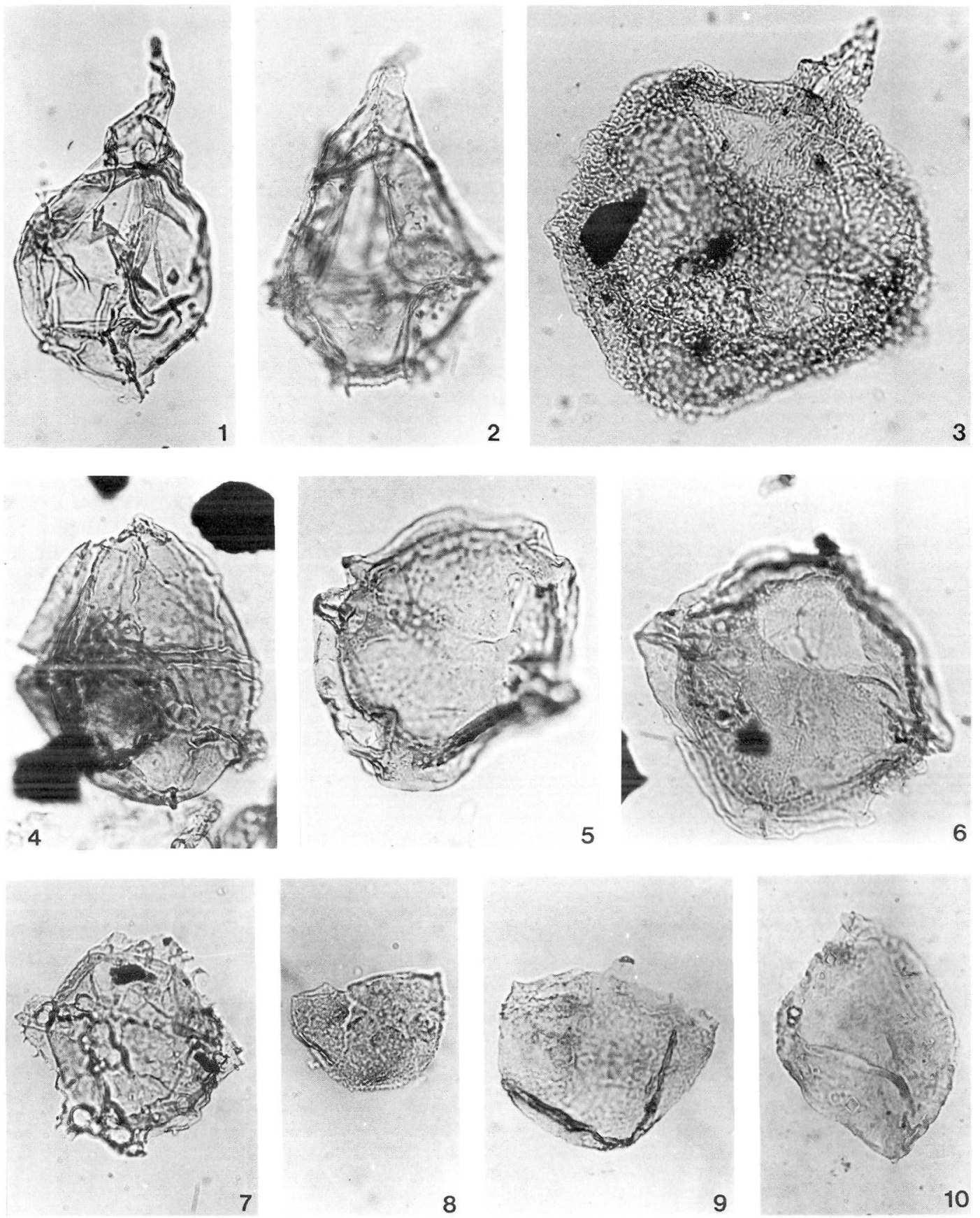

Plate 8. Fig. 1; Gonyaulacysta sp. Hareelv F., (142851, 3-2a). MGUH 16644. Fig. 2; Gonyaulacysta jurassica var. longicornis. Hareelv F., (142844, 3-1). MGUH 16645. Fig. 3; Acanthaulax areolata (Sarjeant) Riley \& Fenton. Hareelv F., (142841, 11-2). MGUH 16646. Fig. 4; Leptodinium sp. Hareelv F., (142855, 3-1). MGUH 16647. Fig. 5; Scriniodinium crystallinum (Deflandre) Klement. Hareelv F., (142849, 4-3). MGUH 16648. Fig. 6; Scriniodinium cf. playfordii Cookson \& Eisenack. Hareelv F., (142849, 3-1). MGUH 16649. Fig. 7; Scriniodinium cf. irregularis (Cookson \& Eisenack) Stover \& Evitt. Hareelv F., (142857, 3-1). MGUH 16650. Fig. 8; Mancodinum semitabulatum Morgenroth. Neill Klinter F., (142829, 4-5). MGUH 16651. Fig. 9. Mancodinium sp. Neill Klinter F., (142829, 4-5). MGUH 16652. Fig. 10; Nannoceratopsis senex van Helden. Neill Klinter F., (142829, 4-2). MGUH 16653. 


\begin{tabular}{lllllllllll}
\hline A1 & A2 & B & (C) & C1 & C2 & C3 & D & D & D & D \\
& & & & & & 44 & 49 & 52 & 58 \\
\hline
\end{tabular}

aff. Baculatisporites sp. 3

Apiculatisporis ovalis

Lycopodiacidites rugulatus

Iraquispora laevigata

Heliosporites altmarkensis

Contignisporites dunrobinensis

Contignisporites glebulensis

Leptolepidites bossus

Manumia cf. irregularis

Contignisporites problematicus

Klukisporites variegatus

Quadraeculina anellaeformis

Staplinisporites caminus

Leptolepidites major

Corollina sp. 1

Sestrosporites pseudoalveolatus

Callialasporites dampieri

Densoisporites velatus

Callialasporites turbatus

Klukisporites pseudoreticulatus

Podocarpidites rousei

Deltoidospora sp. 1

Gleicheniidites conspiciendus

Klukisporites sp. 1

Gleicheniidites senonicus

Baculatisporites sp. 2

Cerebropollenites thiergartii

Spheripollenites subgranulosus

Perinopollenites elatoides

Cerebropollenites macroverrucosus

Baculatisporites comaumensis

Corollina torosus

\begin{tabular}{|c|c|c|c|c|c|c|c|c|c|c|}
\hline $\mathbf{a}$ & + & & & & & & & & & \\
\hline $\mathbf{a}$ & + & & & + & & + & & & & \\
\hline \multirow[t]{6}{*}{$\mathbf{a}$} & & & & & & + & & & & \\
\hline & + & & & & & & & & & \\
\hline & + & & & & & & & & & \\
\hline & & $\begin{array}{l}+ \\
+\end{array}$ & & & & & & & & \\
\hline & & + & & + & & & & & & \\
\hline & & + & & & + & & & & & \\
\hline \multirow[t]{16}{*}{+} & & $\mathbf{a}$ & & + & + & $\mathbf{a}$ & & & & \\
\hline & & $\mathbf{a}$ & & & & + & & + & + & + \\
\hline & & + & & $\mathbf{a}$ & $\mathbf{a}$ & & & & & \\
\hline & & & + & $\mathbf{a}$ & + & & & $t$ & & \\
\hline & & & & + & + & $?$ & & & & \\
\hline & & & & $\mathbf{a}$ & $\mathbf{a}$ & + & & & & + \\
\hline & & & & + & & & + & & & + \\
\hline & & & & + & + & $\mathbf{a}$ & + & & + & + \\
\hline & & & & & + & & & + & + & + \\
\hline & & & & & + & $\mathbf{a}$ & + & & & + \\
\hline & & & & & & + & + & & & \\
\hline & & & & & & + & + & & + & \\
\hline & & & & & & & + & & $\mathbf{a}$ & a \\
\hline & & & & & & & + & & $\mathbf{a}$ & $\mathbf{a}$ \\
\hline & & & & & & & + & + & + & + \\
\hline & & & & & & & & + & & + \\
\hline $\mathbf{a}$ & & & + & + & + & + & & & & + \\
\hline $\mathbf{a}$ & $\mathbf{a}$ & $?$ & & + & + & $?$ & + & + & + & + \\
\hline+ & + & $\mathbf{a}$ & $\mathbf{a}$ & + & + & + & + & + & + & + \\
\hline+ & + & + & + & $\mathbf{a}$ & + & + & + & + & + & + \\
\hline+ & + & + & + & $\mathbf{a}$ & $\mathbf{a}$ & $\mathbf{a}$ & $\mathbf{a}$ & $\mathbf{a}$ & $\mathbf{a}$ & $\mathbf{a}$ \\
\hline $\mathbf{a}$ & + & + & + & $\mathbf{a}$ & + & $\mathbf{a}$ & + & + & + & + \\
\hline $\mathbf{a}$ & & $\mathbf{a}$ & $\mathbf{a}$ & $\mathbf{a}$ & $\mathbf{a}$ & + & + & + & + & + \\
\hline
\end{tabular}

Fig. 5. Stratigraphic distribution of spores and pollen used in defining the assemblages A-D; $a=$ acme. Assemblage $D$ is subdivided and the numbers refer to the uppermost sample of each subdivision.

Subassemblage C1-C2 (Vardekløft Formation, lower part)

The dinoflagellate cyst assemblage with common Sentusidinium pelionense as the only dinoflagellate cyst points in absence of other taxa to a Middle to Late Bajocian sauzei to subfurcatum Zone age for the samples investigated here (Penn, Dingwall \& Knox, 1980; Wollam \& Riding, 1983). The absence of Nannoceratopsis very probably excludes an Early Bajocian to Aalenian, laeviscula Zone, and older age. In Britain Nannoceratopsis is common in the laeviscula Zone and older zones (Penn et al., 1980). The spores and pollen assemblage with Callialasporites and Leptolepidites bossus is only indicative of an age so broadly defined as Late Toarcian to Middle Jurassic. An acme of Perinopollenites elatoides might have correlative value at least for the Vardekløft area. The palynostratigraphy thus provides additional arguments for the hiatus between the Neill Klinter and the Vardekløft Formations suggested by Surlyk et al. 1973.

Subassemblage C3 (Vardekløft Formation, middle and upper part)

The pollen and spores do not indicate any sufficient dating, when compared with the European sections (Couper, 1958, Schulz \& Mai, 1966). The highest occurrence of frequent $\mathrm{BaCu}$ latisporites and Contignisporites can probably be used for regional correlations. The dinoflagellate microflora differs considerably between the two samples investigated. Sample 39 contains a poor microflora with only rare Sentusidinium and $\mathrm{Pa}$ reodinia ceratophora. This is in accordance with 


\begin{tabular}{lllllllllll}
\hline A1 & A2 & B & (C) & Cl & C2 & C3 & D & D & D & D \\
& & & & & & 44 & 49 & 52 & 58 \\
\hline
\end{tabular}

Nannoceratopsis triceras

Veryhachium sortehatense

Nannoceratopsis senex

Mancodinium semitabulatum

Mancodinium sp.

Luehndea spinosa

Nannoceratopsis gracilis

Parvocysta contracta

Sentusidinium pelionense

Ctenidodinium spp.

Carpathodinium predae

Chytroeisphaeridia cerastes

Pareodinia ceratophora

Acanthaulax areolata

Prolixosphaeridium sp.

Adnatosphaeridium aemulum

Gonyaulacysta jurassica v. longicornis

Sentusidinium sp. 1

Cassiculosphaeridia sp.

Endoscrinium galeritum

Sirmiodinium grossii

Meiourogonyaulax sp.

Atopodinium prostatum

Compositosphaeridium costatum

Scriniodinium cf. playfordii

Scriniodinium crystallinum

Hystrichogonyaulax cladophora

Kalyptea cf. glabra

cf. Kalyptea monoceras

Gonyaulacysta sp.

Stephanelytron redcliffense

Leptodinium sp.

Scriniodinium cf. irregularis

Paragonyaulacysta cf. capillosa

cf. Systematophora sp.

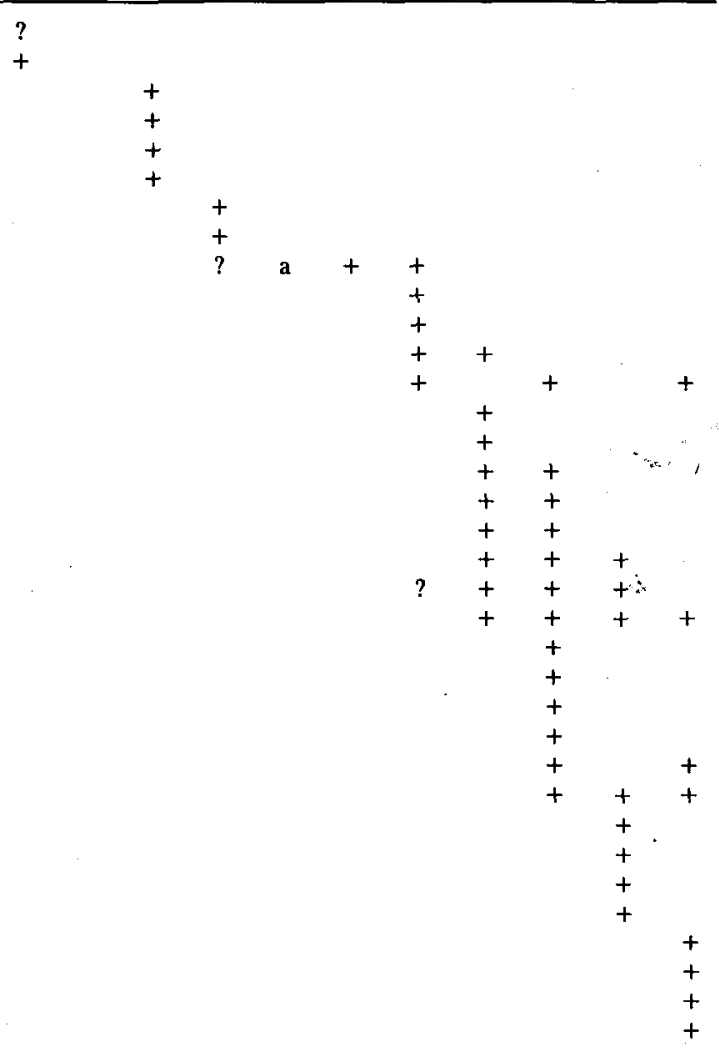

Fig. 6. Stratigraphic distribution of selected dinoflagellate cysts of assemblages A-D; $a=$ acme. Assemblage $D$ is subdivided and the numbers refer to the uppermost sample of each subdivision.

the Bathonian age assigned to this level by regional correlation. In sample 40 a diverse, but only poorly preserved dinoflagellate assemblage with frequent Ctenidonium/Dichadogonyaulax spp. occurs. This frequency suggests an (Early) Callovian or older age (Riley \& Fenton, 1982). Chytroeisphaeridia cerastes is present and indicates compared with Britain an age not older than the latest Early Callovian, calloviense calloviense ammonite Subzone (Riley \& Fenton, 1982). Carpathodinium predae which was described from undifferentiated Callovian to Early Oxfordian of Rumania is in view of its to Bathonian restricted occurrence in England (Wollam \& Riding, 1983), considered to indicate (Early) Callovian to Bathonian. An (Early) Callovian age is thus probable for sample 40 .

\section{Assemblage D (lower Hareelv Formation)}

Gleicheniidites conspiciendus which is the main spore to characterize assemblage D occurs as early as Middle Bajocian in Europe (Schulz \& Mai, 1966). In East Greenland it has not been observed below the Oxfordian (dated by ammonites and dinoflagellate cysts), in N. Norway it was not observed below the Early Kimmeridgian (Vigran \& Thusu, 1975) and on Svalbard it has not yet been recorded.

Age significant correlation based on spores and pollen of the assemblage $D$ microflora appears impossible, probably due to palaeolatitudal variations as indicated by the first occurrence of G. conspiciendus. However, the dinoflagellate cysts allow reliable datings as discussed below. 
Samples 41-45 (assemblage D, lower part)

The deepest part investigated of the Hareelv Formation (samples 41-45) yielded a considerable number of the morphologically very characteristic Acanthaulax areolata. In Britain this species is known from the Late Callovian, athleta Zone to the Middle Oxfordian, plicatilis Zone with a maximum in the Early Oxfordian (Sarjeant, 1979, for Gonyaulacysta scarburghensis; Riley \& Fenton, 1982, for A. senta). Wanaea spp. were not found; this probably excludes the mariae and lamberti Zones. Another dinoflagellate species of prime stratigraphic importance is Adnatosphaeridium aemulum (base sample 43) which according to Piasecki (1980) in East Greenland is restricted to the Early Oxfordian, cordatum Zone - Middle Oxfordian, basal tenuiserratum Zone with acme in the late cordatum Zone. Sentusidinium sp. 1 also excludes a pre cordatum Zone age. The concurrent presence of $A$. areolata and $A$. aemulum thus dates this assemblage to the Early Oxfordian, cordatum Zone to Middle Oxfordian, plicatilis Zone. Within this time range the considerable frequency of $A$. areolata favours a cordatum Zone age.

\section{Samples 47-52 (assemblage D, middle part)}

In sample 47 a rich dinoflagellate cysts assemblage with 26 species was observed. Noteworthy is the incoming of Hystrichogonyaulax cladopho$r a$ (frequent) and of Scriniodinium crystallinum and $S$. cf. playfordii. A. aemulum is still frequent whereas $A$. areolata is missing. Atopodinium prostatum and Meiourogonyaulax sp. both are of stratigraphic interest. A. prostatum is hitherto known from the Late Callovian, athleta Zone to Early Oxfordian, cordatum Zone (Riley \& Fenton, 1982) whereas Meiourogonyaulax sp. was found by Piasecki (1980) only in the Middle Oxfordian lower tenuiserratum Zone. Obviously at least one of these two ranges mentioned is too short. A Middle Oxfordian, probably densiplicatum/plicatilis Zone age is proposed for sample 47 due to frequent $A$. aemulum, presence of $A$. prostatum and absence (?low frequency) of $A$. areolata. A Middle Oxfordian age also apply for sample 49 where $A$. aemulum is still frequent and where Compositosphaeridium costatum occurs.
Gonyaulacysta jurassica var. longicornis and $A$. aemulum have their tops in sample 49.

In samples 51 and 52 a rather poor assemblage of dinocysts was found. It is characterized by $\mathrm{Go}$ nyaulacysta sp. and the last occurrence of Endoscrinium galeritum. A Late Oxfordian age is suggested for this thin interval by the absence of older taxa and the presence of $E$. galeritum, which in this region has its highest occurrence in the Late Oxfordian, glosense Zone (Piasecki, 1980).

\section{Sample 55-58 (assemblage D, upper part)}

In sample 55 the dinoflagellate cyst assemblage is characterized by Leptodinium sp. (common) which is interrelated to Leptodinium sp. A of Bjærke, known from the Late Oxfordian to Early Kimmeridgian of Svalbard (Bjærke, 1977).

In sample 56 a cavate variety of this Leptodinium sp. occurs. Due to this and to the absence of $E$. galeritum, sample 55 and 56 are considered to be of Late Oxfordian, probably post glosense Zone to Early Kimmeridgian age.

In sample 57 and 58 an Early Kimmeridgian age is indicated by the concurrent presence of Scriniodinium cf. irregularis and Scriniodinium crystallinum which in East Greenland occur together only in the Early Kimmeridgian baylei to eudoxus Zone (Piasecki, 1980).

The palynological correlations suggested for the Neill Klinter and Vardekløft Formations are in agreement with the ages based on the marine fauna proposed by Surlyk et al. (1973). The study of the palynomorphs also confirms the presence of a hiatus between the two formations corresponding to the time interval from the Aalenian to at least the Early Bajocian, laeviscula Zone.

In the present study the lower part of the Hareelv Formation has been referred to the Early (-Middle) Oxfordian cordatum (-plicatilis) Zone. This corresponds approximately to the age of the Olympen Formation of northern Jameson Land which based on ammonite correlations was determined as Middle Oxfordian plicatilis Zone and older (Surlyk et al., 1973). Thus the deeper part of the Harreelv Formation may be a southern clayey equivalent to the sandy Olympen Formation in the north as also recognized by Surlyk (1977, Table 2). 


\section{Comparison with Svalbard}

The Upper Triassic Norian to Rhaetian palynostratigraphy of Svalbard was compared with East Greenland by Raunsgaard Pedersen \& Lund (1980). The Jurassic is compared below.

The oldest Jurassic microflora known from Svalbard contains Cerebropollenites mesozoicus and Limbosporites lundbladii. Bjærke (1977) found this flora in the upper part of the Wilhelmøya Formation (association $\mathrm{C}$ of Bjærke) and suggested an Early Jurassic, Sinemurian age. Thus it probably falls within the hiatus between the Kap Stewart Formation and the Neill Klinter Formation.

A microflora of late Early Jurassic, Pliensbachian to Toarcian age was found just below the Brentskardhaugen Bed in Sassenfjord, Spitsbergen (Bjærke \& Dypvik, 1977). The microflora of these strata comprises Nannoceratopsis, Klukisporites variegatus, Contignisporites dunrobinensis, etc. indicating a correlation with the Neill Klinter Formation (above sample 27) in East Greenland. The characteristic acme of Spheripollenites (assemblage B) is not recorded in Svalbard.

A rich dinoflagellate microflora occurs in the Toarcian phosphoritic pebbles of the Brentskardhaugen Bed at the base of the Middle to Upper Jurassic Agardhfjellet Formation/Member (Bjærke, 1980a). Parvocysta contracta is present and suggests a correlation with sample 31 of the uppermost Neill Klinter Formation. No details of the spore pollen microflora have been published from this level in Svalbard.

The Middle to Upper Jurassic microfloras of the Agardhfjellet Formation/Member are known from Kong Karls Land (Bjærke, 1977; Thusu, 1978) and Spitsbergen (Bjærke, 1980b). At the base of the Agardhfjellet Formation/Member, Bjærke (1977) described a faunally dated Callovian microflora (association D of Bjærke) with the dinoflagellate cysts Sirmiodinium grossii, Pareodinia spp., Gonyaulacysta jurassica etc. and the spore-pollen species Contignisporites problematicus, Quadraeculina anellaeformis (al. Ovalipollis limbata), Corollina sp. 1 (al. C. torosus) etc. Compared with this study, the presence of $S$. grossii suggests a correlation with the Hareelv Formation. The spore-pollen species, however, suggest affinities with the Vardekløft Formation.
The age of the basal Agardhfjellet Formation probably thus falls within the time span between the youngest Vardekløft and the oldest Hareelv Formation samples investigated here. Fensome (1979) found $S$. grossii upwards from the upper Vardekløft Formation. Together with the spores and pollen mentioned this indicates that the basal Agardhfjellet correlates with the upper Vardekløft Formation.

An Upper (to Middle) Callovian dinocyst microflora was described from the lower part of the Agardhfjeller Formation/Member in Spitsbergen by Bjærke (1980b). This microflora (Bjærke's Zone 2) which contains Pareodinia prolongata, Wanaea sp. and Acanthaulax areolata (al. A. senta) falls within the hiatus between the Vardekløft and the Harrelv Formations as studied here.

Bjærke (1977) described an Oxfordian/Kimmeridgian microflora higher in the Agardhfjellet Formation/Member (named assemblage E) with Leptodinium sp. A, Gonyaulacysta jurassica var. longicornis and cf. Systematophora sp. (al. Sp. Indet). This microflora points to an approximate correlation with sample 55 from the Late Oxfordian to Early Kimmeridgian of the Hareelv Formation.

In the upper part of the Agardhfjellet Formation Bjærke (1980b) described a marine microflora (his Zone 3) which is typified by Paragonyaulacysta and Horologinella spinosigibberosa. This microflora suggests a correlation with the Lower Kimmeridgian sample 57/58 of the Harreelv Formation or with higher Jurassic strata not exposed in the Vardekløft profile.

\section{Comparison with Andøya}

About $350 \mathrm{~m}$ of Middle and Upper Jurassic sediments occur on the Norwegian island of Andøya which during the Jurassic was located about midway between Scoresby Sund and Svalbard. The section comprises the Ramså Formation of Bajocian to ?Oxfordian age (Vigran \& Thusu, 1975) and the Dragneset Formation, from which Early Kimmeridgian, Middle Volgian and Early Cretaceous ammonites are known (Birkelund, Thusu \& Vigran, 1978).

The lower part of the Ramsa Formation with the Hestberget Member and Kulgrøfta Member 
contains a rich non-marine microflora with $\mathrm{Cal}$ lialasporites (al. Aplanopsis), Contignisporites problematicus (al. Corrugatisporites amplectiformis), Lycopodiacidites rugulatus; Gleicheniidites conspiciendus is absent (Vigran \& Thusu, 1975). This microflora is thus typical of an assemblage $\mathrm{C}$ type and indicates a correlation with the Vardekløft Formation. Podocarpidites rousei points to a correlation with the Callovian part of the Vardekløft Formation (subassemb. C3 microflora). However, the presence of Gleicheniidites senonicus, observed only in the assemblage D of East Greenland, favours a somewhat younger age. A Callovian age is therefor proposed for the Hestberget and Kulgrøfta Member.

The lower part of the Dragneset Formation with the Breisanden Member, dated as Early Kimmeridgian by ammonites (cymodoce Zone), contains a microflora with spores, pollen and dinoflagellate cysts (Vigran \& Thusu, 1975; Birkelund et al., 1978). Gleicheniidites conspiciendus is present indicating a correlation with the Hareelv Formation as exposed in the Vardekløft profile. Cicatricosisporites sternum was noted by Vigran \& Thusu, 1975, showing that this characteristic species and representative of a mainly Early Cretaceous genus occurs earlier in the north than in Central Europe.

\section{Palaeoenvironment}

The relative occurrence of the main morphological groups of spores and pollen and of dinoflagellate cysts in the Neill Klinter, Vardekløft, and lower Hareelv Formations used for the interpretation of the palaeoenvironment is shown in figure 7. The diagram is based on counts of $200-300$ non-fragmented specimens from selected samples of well-preserved material. It was constructed according to the methods used by Pedersen \& Lund (1980) for the underlying Kap Stewart Formation which was studied from exposures in Astartekløft about $2 \mathrm{~km}$ north of Vardekløft.

All microfloras studied show predominance of terrestrial derived palynomorphs relative to marine palynomorphs.

The trilete spores (column A fig. 7) vary from 15 to 45 per cent with maximum occurrence both in the lower part of the section (sample 23, subassemb. A1) and in the upper middle part (sample
39-40, subassemb. C3). The laevigate trilete spores (indicated by the open signature in column $\mathrm{A}$ ) show less frequency variations than the sculptured trilete spores. The monolete spores (column B) are rare in all samples. The saccate pollen (column $C$ ) show a maximum occurrence in the lower part of section (sample 27, subassemb. A2) where they comprise up to 55 per cent of the specimens. They occur infrequently in the middle part, but register percentages of up to 40 per cent in the upper part of the section (sample 40-58, subassemb. C3, assemblage D). Vitreisporites, indicated in the column $\mathrm{C}$ by the closer signature, show higher values at the top of the profile (samples 43-58).

The aperturate pollen (column D) generally show low values, but with an extreme maximum of 80 per cent in the lower part of the section (sample 28, assemblage B) and up to 30 per cent in the upper part (sample 51-53, assemblage D). The maximum in assemblage $B$ is due to the mass occurrence of Spheripollenites, and the maximum in assemblage $D$ represents common monosulcate pollen including Monosulcites, which is shown with closer signature in column D. Corollina is rare (less than $2 \%$ except in sample 28 where it comprises up to $6.2 \%$ ). In the same sample disaccate conifer pollen are almost absent.

A similar maximum of Spheripollenites was noted by Morbey (1978) in NW Europe and could be climatic controlled. This coincides with a maximum of Corollina and a minimum of disaccate pollen.

The alete/inaperturate pollen (column E) are most abundant in the middle of the section where they comprise up to 40 per cent of the specimens counted (sample 33-38, subassemb. C1 and C2), and in the upper part of the section (sample 4358 , assemblage $\mathrm{D}$ ).

The marine palynomorphs (column F) generally form only a minor part of the microfloras constituting from about 2 per cent of the specimens in the lowermost part of the section (sample 23, subassemb. A1, with acritarchs) and about 3 per cent in the middle part (sample 39, subassemb. C3) to as much as 10 per cent in the upper part of the section (sample 43, lower assemblage $\mathrm{D}$, mainly with dinoflagellate cysts). The highest values of the dinocysts concur with relatively high numbers of saccate pollen and moderate numbers of spores. The lowest num- 


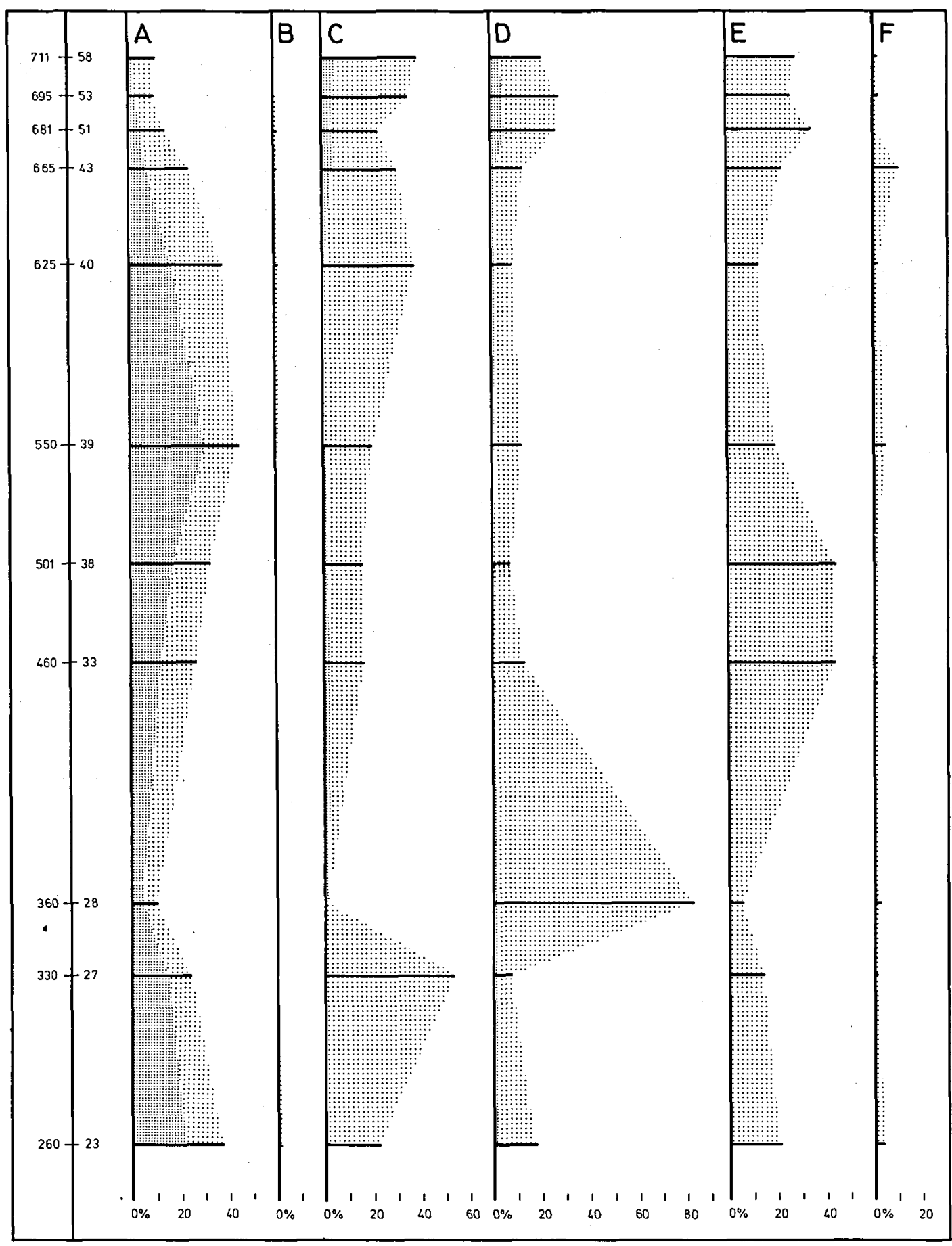

Fig. 7. Diagram showing the relative occurrence of the main morphological groups of spores and pollen and dinoflagellate cysts in selected samples from the marine formations in the Vardekløft ravine. A: trilete, B. monolete, C: saccate, D: monocolpate, E: alete, F: dinoflagellate cysts. Column $A$ is subdivided into two, the open signature indicating laevigate trilete spores; and the closer signature sculptured trilete spores. In column $\mathbf{C}$ the close signature represents Vitreisporites and in column D Monosulcites. The thick lines represent the samples counted. For these, sample numbers (only last two numbers) and meters above sea level are shown on the left. 
bers of marine cysts are in the Sorthat Member of the Vardekløft Formation (sample 33, subassemb. C1).

The amount of trilete spores is important for the interpretation of the the palaeoenvironment. This group comprises more heavy, mainly water transported, palynomorphs, and its frequency is probably related to distance to rivers and shorelines. A maximum of trilete spores occurs in the lower part of the Neill Klinter Formation and in the middle to upper Vardekløft Formation. The relative occurrence of the main palynomorph groups with variation and predominance of terrestrially derived forms generally indicate a nearshore depositional environment. The distance to land probably reached its maximum within the Neill Klinter Formation (sample 27, subassemb. A2) as shown by the maximum of disaccate pollen and low diversity of the other groups. In samples 51-58 from the Hareelv Formation the pollen suited for wind transportation are exceptionally varied with Cerebropollenites, monosulcate and disaccate pollen (including a maximum of $\mathrm{Vi}$ treisporites) occurring frequently. This coincides with a minimum of spores and thus suggests an environment, with little fluviatile influence, but rather close to the shore.

The freshwater algae Botryococcus is common in the lower Vardekløft Formation (samples 3235). In the Middle Jurassic Brent Formation of the northern North Sea such occurrences were restricted to the pro-delta clays (Parry, Whitely \& Simpson, 1981). The microflora seems to be in accordance with this environment and the associated dinoflagellate cysts assemblage is monotypic, suggesting restricted conditions, perhaps relating to a brackish subbasin.

Acknowledgements. The field work was partly financed by the Carlsberg Foundation and by the Geological Survey of Greenland (G.G.U.). The laboratory work has been carried out at the Section for Palynology, Geological Institute, University of Aarhus.

We wish gratefully to thank B. Larsen, L. Eklund-Jürgensen, M. Dybdahl, L. Jans and J. Sommer for their work with preparations, typing, drawing and photography in connection with the project. E. M. Friis is thanked for valuable help. S. Piasecki, G.G.U. is thanked for constructive comments. R. E. Dunay, Mobil, Celle kindly improved the English manuscript.

Deutsche Texaco AG and Texaco Inc. are thanked for permission to J. J. Lund to publish this paper.

The results on G.G.U. material are published with permission of the Geological Survey of Greenland.

\section{Dansk sammendrag}

De marine jurassiske formationer: Neill Klinter, Vardekløft og nedre Hareelv Formation er blevet palynologisk undersøgt på grundlag af materiale fra blotninger i Vardekløft i det østlige Jameson Land.

Den unders $ø$ gte lagserie er ca. $500 \mathrm{~m}$ tyk, men stedvis dảrlig blottet. Undersøgelsen repræsenterer en fortsættelse af tidligere publicerede profiler $i$ den underliggende limniske Kap Stewart Formation, der blev henført til nedre rhaetien og hettangien.

De undersøgte mikrofloraer omfatter talrige sporer og pollen, samt dinoflagellat-cyster. På grundlag af forekomsten af spore-pollen arterne er der foretaget en opdeling $i$ fire selskaber (assemblage A-D). Der er foreslået biostratigrafiske korrelationer, også støttet af dinoflagellat-arterne, ved sammenligning med mikrofloraer fra Europa fra nedre, mellem og øvre jura.

Assemblage A og B, der forekommer i Neill Klinter Formationen, er blevet korreleret til ( $\varnothing \mathrm{vre}$ ) pliensbachien til nedre toarcien. Den typiske assemblage $\mathrm{C}$ mikroflora forekommer $\mathrm{i}$ Vardekløft Formationen og er henført til mellem-øvre bajocien ved basis af formationen og til (nedre) callovien фverst $i$ formationen. Assemblage D mikrofloraen forekommer $\mathrm{i}$ den nedre del af Harrelv Formationen og er på grundlag af dinoflagellat-arterne henført til oxfordien (cordatum(-plicatilis) Zonen) ved basis af formationen, og til nedre kimmeridgien i den øverste del af profilet. De palynologiske korrelationer for Neil Klinter og Vardekløft Formationerne er i overensstemmelse med de aldre, der er foreslået udfra de marine faunaer. Resultaterne bekræfter tilstedeværelsen af en hiatus mellem de to formationer, der omfatter tidsintervallet fra aalenien til idet mindste nedre bajocien.Den nedre del af Hareelv Formationen henføres til nedre (-mellem) oxfordien. Dette svarer nasten til alderen på Olympen Formationen fra det nordlige Jameson Land.

Sammensætningen af mikrofloraerne viser hen til marine til brakiske aflejringsforhold. Der er dog en klar dominans af de palynomorf-grupper, der hidrører fra landvegetationen i forhold til de marine palynomorfer. Udfra de betydelige variationer, der konstateres i palynomorf-materialet, der hidrører fra landområderne, vurderes aflejringsforholdene nøjere.

\section{References}

Alberti, G. 1961: Zur Kenntnis mesozoischer und alttertiärer Dinoflagellaten und Hysterichosphaerideen von Nord- und Mitteldeutschland, sowie einigen anderen europäischen Gebieten. Palaeontographica, Abt A 116, 1-58.

Balme, B. E. 1957: Spores and Pollen Grains from the Mesozoic of Western Australia. Coal Res. C.S.I.R.O. Ref. T.C. $25,1-48$.

Below, R. 1982: Rigaudella, ein neues Genus von Dinoflagellaten-Zysten. N. Jb. Geol. Palönt. Mh. 3, 137-150.

Birkelund, T., Thusu, B. \& Vigran, J. 1978: Jurassic-Cretaceous biostratigraphy of Norway, with comments on the British Rasenia cymodoce Zone. Palaeontology 21(1), 31-63.

Bjærke, T. 1977: Mosozoic Palynology of Svalbard - II. Palynomorphs from the Mesozoic sequence of Kong Karls Land. Norsk Polarinst. Arbog 1976, 83-120.

Bjærke, T. 1980a: Mesozoic Palynology of Svalbard - IV. Toarcian dinoflagellates from Spitsbergen. Palynology 4, 5777.

Bjærke, T. 1980b: Mesozoic Palynology of Svalbard - V. Dinoflagellates from the Agardhfjellet Member (Middle and Upper Jurassic) in Spitsbergen. Norsk Polarinst. 172, 145167. 
Bjærke, T. \& Dypvik, H. 1977: Sedimentological and palynological studies of Upper Triassic-Lower Jurassic sediments in Sassenfjorden, Spitsbergen. Norsk Polarinst. Arb. 1976, 131-150.

Cookson, I. C. 1947: Plant microfossils from the lignites of Kergulen Archipelago. B.A.N.Z. antarc. Res. Exped. 19291931, Rep. Ser. A.II 8, 127-142.

Cookson, I. C. \& Eisenack, A. 1960: Upper Mesozoic microplankton from Australia and New Guinea. Palaeontology 2, 243-261.

Couper, R. A. 1958: British Mesozoic microspores and pollen grains. A systematic and stratigraphic study. Palaeontographica, Abt B 103(4-6), 75-179.

Danzé-Corsin, P. \& Laveine, J.-P. 1963: Flore infraliassique du Boulonnais. B. Microflore. Mém. Soc. Géol. Nord. 13, 57-143.

Davey, R. J. 1969: Non-calcareous microplankton from the Cenomanian of England, northern France and North America, Part I. Bull. Brit. Mus. (Nat. Hist.) Geol. 17, 103-180.

Davey, R. J. 1979: A Re-appraisal of the Genus Chytroeisphaeridia Sarjeant 1962. Palynology 3, 209-218.

Deflandre, G. 1938: Microplancton des mers Jurassiques conservé dans les marnes de Villers-sur-Mer (Calvados) Étude liminaire et considérations générales. Travaux de la Station Zoologique de Wimereux 13, 147-200.

Deflandre, G. 1947: Sur quelques micro-organismes plantoniques des silex Jurassiques. Bulletin de L'Institut Océanographique de Monaco 921, 1-10.

Dettmann, M. E. 1963: Upper Mesozoic microfloras from South-Eastern Australia. Proc. R. Soc. Vic., N.S. 77(1), $1-148$.

Dev, S. 1961: The Fossil Flora of the Jabalpur Series - 3. Spores and Pollen Grains. Palaeobotanist 8(1), 43-56.

Dodekova, L. 1974: Compositosphaeridium gen. n. - a new genus dinoflagellate cyst. Bulgarian Acad. Sci., Comm. Géol. Bull. Géol. Inst., Sér. Paléont. 23, 25-30.

Dodekova, L. 1975: New Upper Bathonian dinoflagellate cysts from north-eastern Bulgaria: Bulgarian Acad. Sci., Palaeo. Strat. and Lith. 2, 17-34.

Downie, C. \& Sarjeant, W.A.S. 1963: On the interpretation and status of some hystrichosphere genera. Palaeontology 6, 83-96.

Downie, C. \& Sarjeant, W.A.S. 1964: Bibliography and index of fossil dinoflagellates and acritarchs. Geol. Soc. Am. Mem. 94, 1-180.

Drugg, W. S. 1978: Some Jurassic dinoflagellate cysts from England, France and Germany, Palaeontographica Abt. B $168,61-79$.

Döring, H. 1965: Die sporenpaläontologische Gliderung des Wealden in Westmecklenburg (Struktur Werle). Geologie 14(47), 1-118.

Erdtman, G. 1948: Did dicotyledonous plants exist in Early Jurassic times? Geol. För. Stockh. Förh. 70, 265-271.

Erkmen, U. \& Sarjeant, W.A.S. 1980: Dinoflagellate cysts, acritarchs and tasminitids from the uppermost Callovian of England and Scotland: With a reconsideration of the "Xanthidium pilosum" problem. Geobios 13, 45-99.

Fensome, R. A. 1979: Dinoflagellate cysts and acritarchs from the Middle and Upper Jurassic of Jameson Land, East Greenland. Bull. Gronlands geol. Unders. 132, 1-98.

Harris, T. M. 1937: The fossil flora of Scoresby Sound East Greenland. 5. Stratigraphic relations. Meddr. Grønland. 112(2), 1-114.

Ioannides, N. S., Stavrinos, G. N. \& Downie, C. 1977: Kimmeridgian microplankton from Clavel's Hard, Dorset, England. Micropaleontology 22, 443-478.

Kedves, M. \& Simoncsics, P. 1964: Microstratigraphy of the carbonate manganese ore layer of Shaft III of Urkút on the basis of palynological investigations. Acta Min. Pet. 16 (2), 3-48.
Kemp, E. M. 1970: Aptian and Albian miospores from southern England. Palaeontographica, Abt. B 131(1-4), 73-143.

Klement, K. W. 1960: Dinoflagellaten und Hystrichosphaerideen aus dem unteren und mittleren Malm Südwestdeutschlands. Palaeontographica, Abt. A 114, 1-104.

Klaus, W. 1960: Sporen der Karnischen Stufe der ostalpinen Trias. Jahrb. Geol. Bundesanst., Sonderb. 5, 107-183.

Krutzsch, W. 1959: Mikropaläontologische (sporenpaläontologische) Untersuchungen in der Braunkohle des Geiseltales. Geologie 8 (21-22), 1-425.

Lund, J. J. 1977: Rhaetic to Lower Liassic palynology of the onshore south-eastern North Sea Basin. Danm. geol. Unders., (2) 109, 1-103.

Maljavkina, V. S. 1949: Diagnose der Sporen und Pollen von Jura und Kreide. Tr. VNIGRI 33, 1-137 (in Russian).

Morbey, S. J. 1978: Late Triassic and Early Jurassic Subsurface palynostratigraphy in Northwestern Europe. Palinologia, núm. extraord. 1, 355-368.

Morgenroth, P. 1970: Dinoflagellate cysts from the Lias Delta of Lühnde/Germany. N. Jb. Geol. Paläont., Abh. 136, 345-359.

Mädler, K. 1964: Die geologische Verbreitung von Sporen und Pollen in der deutschen Trias. Beih. Geol. Jahrb. 65, 1147.

Nilsson, T. 1958: Über das Vorkommen eines mesozoischen Sapropelgesteins in Schonen. Lunds Univ. Ársskr., N. F. Avd. 2, 54(10), 1-111.

Norris, G. 1965: Triassic and Jurasic miospores and acritarchs from the Beacon and Ferrar Groups, Victoria Land, Antarctica. N.S.J. Geol. Geophys. 8(2), 236-277.

Norris, G. \& Sarjeant, W.A.S. 1965: A descriptive index of genera of fossil Dinophyceae and Acritarcha. N.Z. Geol. Surv. Paleont. Bull. 40, 1-72.

Parry, C. C., Whitely, P. K. J. \& Simpson, R. D. H. 1981: Integration of palynological and sedimentological methods in facies analysis of the Brent Formation. In L. V. Illing \& G. D. Hobson (eds.): Petroleum Geology of the Continental Shelf of North-West Europe, 205-215. Institute of Petroleum, London.

Penn, I. E., Dingwall, R. G. \& Knox, R. W. O'B. 1980: The Inferior Oolite(Bajocian) sequence from a borehole in Lyme Bay, Dorset. Inst. Geol. Sci. Report 79/3, 1-27.

Pedersen, K. R. \& Lund, J. J. 1980: Palynology of the plantbearing Rhaetian to Hettangian Kap Stewart Formation, Scoresby Sund, East Greenland. Rev. Palaeobot. Palynol. $31,1-69$.

Pflug, H. 1953: Zur Entstehung und Entwicklung des angiospermiden Pollens in der Erdgeschichte. Palaeontographica Abt. B. 95(4-6), 60-171.

Piasecki, S. 1980: Middle to Late Jurassic dinoflagellate cyst stratigraphy from Milne Land and Jameson Land (East Greenland) correlated with ammonite stratigraphy. Unpubl. thesis, University of Copenhagen.

Pocock, S. A. J. 1962: Microfloral Analysis and Age Determination of the strata at the Jurassic-Cretaceous Boundary in the Western Canada Plains. Palaeontographica, Abt. B. 111(1-3), 1-95.

Pocock, S. A. J. 1970: Palynology of the Jurassic Sediments of Western Canada. Part 1. Terrestrial species. Palaeontographica, Abt. B. 130(1-2): 12-72, continued in (3-6), 73136.

Potonié, R. 1956: Synopsis der Gattungen der Sporae dispersae. I Teil: Sporites. Beih. Geol. Jb. 23, 1-103.

Reinhardt, P. 1961: Sporae dispersae aus dem Rhät Thüringens. Mbr. dt. Akad. Wiss. Berl., 704-711.

Riley, L. A. \& Fenton, J. P. G. 1982: A Dinocyst zonation for the Callovian-Middle Oxfordian succession of Northwest Europe. Palynology 6, 193-202.

Ross, N. E. 1949: Investigations of the Senonian of the Kristianstad District, S. Sweden. I: On a Cretaceous Pollen 
and Spore Bearing Clay Deposit of Scania. Bull. geol. Instn, Univ. Upsala XXXIV, 25-43.

Sarjeant, W. A. S. 1961: Microplankton from the Kellaways Rock and Oxford Clay of Yorkshire. Palaeontology 4, 90118.

Sarjeant, W. A. S. 1962: Microplankton from the Ampthill Clay of Melton, south Yorkshire. Palaeontology 5, 478497.

Sarjeant, W. A. S. 1969: Taxonomic changes. In Davey, R. J., Downie, C., Sarjeant, W. A. S. \& Williams, G. L.: Appendix to "Studies on Mesozoic and Cainozoic dinoflagellate cysts". Bull. Brit. Mus. (Nat. Hist.) Geol., Appendix to Supplement 3, 7-15.

Sarjeant, W. A. S. 1979: Middle and Upper Jurassic Dinoflagellate Cysts: The World excluding North America. AASP Contrib. Ser. 5B, 133-151:

Sarjeant, W. A. S. \& Stover, L. E. 1978: Cyclonephelium and Tenua, a problem in dinoflagellate cyst taxonomy. Grana $17,47-58$.

Schulz, E. 1962: Sporenpaläontologische Untersuchungen zur Rhät-Lias-Grenze im Thüringen der Altmark. Geologie 11(3), 308-319.

Schulz, E. 1966: In Döring, H., Krutzsch, W., Schulz, E., \& Timmermann, E.: Über einige neue Subformgenera der Sporengattung Stereisporites Th. \& Pf. aus dem Mesozoicum und Alttertiär Mitteleuropas. Beih. Geol. 55, 72-89.

Schulz, E. 1967: Sporenpaläontologische Untersuchungen rhätoliassischer Schichten im Zentralteil des Germanischen Beckens. Paläontol. Abh. B 2(3), 541-633.

Schulz, E. \& Mai, D. H. 1966: Erläuterungen zur Tabelle der stratigraphischen Verbreitung des Phytoplanktons im Lias und Dogger. In Döring, H., Krutzsch, W., Mai, D. H. \& Schulz, E: Erläuterungen zu den sporenstratigraphischen Tabellen vom Zechstein bis zum Oligocän. Abh. Zentr. Geol. Inst. 8, 35-45.

Stover, L. E. \& Evitt, W. E. 1978: Analyses of pre-Pleistocene organic-walled dinoflagellates. Stanford Univ. Publ., Geol. Sci. 15, 1-300.

Surlyk, F. 1977: Stratigraphy, tectonics and palaeogeography of the Jurasic sediments of the areas north of Kong Oscars
Fjord, East Greenland. Bull. Grønlands geol. Unders. 123, 1-56.

Surlyk, F., Bromley, R. G., Asgaard, U. \& Pedersen, K. R. 1971: Preliminary account of mapping of the Mesozoic Formations of south-east Jameson Land. Rapp. Grønlands geol. Unders. 37, 24-32.

Surlyk, F., Callomon, J. H., Bromley, R. G. \& Birkelund, T. 1973: Stratigraphy of the Jurassic-Lower Cretaceous sediments of Jameson Land and Scoresby Land, East Greenland. Bull. Grønlands geol. Unders. 105, 1-76.

Thusu, B. 1978: Distribution of biostratigraphically diagnostic dinoflagellate cysts and miospores from the Northwest European continental shelf and adjacent areas. Continental Shelf Institute Publ. 100, 1-111.

Tralau, H. 1968: Botanical Investigations into the Fossil Flora of Eriksdal in Fyledalen, Scania. II: The Middle Jurassic Microflora. Sver. Geol. Unders. Afh. ser. C. 633, 1-185.

Van Helden, B. G. T. 1977: Correlation of microplankton assemblages with ammonite faunas from the Jurassic Wilkie Point Formation, Prince Patric Island, District of Franklin. Geol. Surv. Canada, Paper 77-LB, 163-171.

Vigran, J. O. \& Thusu, B. 1975: Illustrations and Distribution of the Jurassic Palynomorphs of Norway. Roy. Norw. Counc. Sci. Ind. Res., Cont. Shelf Div. Publ. 65, 1-55.

Weyland, H. \& Krieger, W. 1953: Die Sporen und Pollen der Aachener Kreide und ihre Bedeutung für die Charakteriserung des mittleren Senons. Palaeontographica (Abt. B) 95, 6-29.

Wiggins, V. D. 1975: The dinoflagellate family Pareodiniaceae: a discussion. Geoscience and Man 11, 95-115.

Wille, W. 1982: Evolution and ecology of Upper Liassic dinoflagellates from SW Germany. N. Jb. Geol. Paläont. Abh. 164(1-2), 74-82.

Williams, G. L. \& Downie, C. 1969: Generic re-allocations. In Davey, R. J., Downie, C., Sarjeant, W. A. S. \& Williams, G. L.: Appendix to "Studies on Mesozoic and Cainozoic dinoflagellate cysts". Bull. Brit. Mus. (Nat. Hist.) Geol., Appendix to Supplement 3, 1-17.

Wollam, R. \& Riding, J. B. 1983: Dinoflagellate cyst zonation of the English Jurassic. Inst. geol. Sci. Report 83/2, 42 pp. 